\title{
Static Analytical Approach of Moderately Thick Cylindrical Ribbed Shells Based on First-Order Shear Deformation Theory
}

\author{
Jinxing Lai, ${ }^{1}$ Chunxia Guo, ${ }^{2}$ Junling Qiu, ${ }^{1}$ and Haobo Fan ${ }^{1}$ \\ ${ }^{1}$ School of Highway, Chang'an University, Xian 710064, China \\ ${ }^{2}$ School of Science, Xian University of Architecture and Technology, Xian 710055, China \\ Correspondence should be addressed to Chunxia Guo; 1006707701@qq.com
}

Received 22 December 2014; Revised 12 February 2015; Accepted 12 February 2015

Academic Editor: Francesco Tornabene

Copyright (c) 2015 Jinxing Lai et al. This is an open access article distributed under the Creative Commons Attribution License, which permits unrestricted use, distribution, and reproduction in any medium, provided the original work is properly cited.

\begin{abstract}
The classical shell theory (CST) without considering the shear deformation has been commonly used in the calculation of shells structures recently. However, the impact of theory of plates and shells subjected to the shear deformation on the calculation is increasingly pronounced along with the wide use of composite laminated structures. In this paper, based on first-order shear deformation theory (FSDT) of cylindrical shells, the displacement control differential equation of moderately thick cylindrical shells has been obtained, so has been the edge force at longitudinal of the shells. Meanwhile, a group of unit force is introduced to deduce the displacement of edge beam under the action of edge force. A join condition of moderately thick cylindrical ribbed shells is established according to the continuity of displacement as well. Most notably, the displacement analytical solution of bending problems of moderately thick cylindrical ribbed shells is obtained, which has profound theoretical significance for further improving the analytical solution of moderately thick cylindrical shells.
\end{abstract}

\section{Introduction}

With the advent of new materials, various shell structures have experienced unprecedented development. In comparison with the beam and plate structural and the spherical shell structure, the cylindrical shell is of more reasonable structure, superior mechanical performance, simpler construction, and manufacturing. Therefore, the cylindrical shells have been widely used in tunnel, subway, and pipe jacking and other municipal engineering and machinery and aviation industries $[1,2]$. Because of the engineering design requirement, the thickness of shells structures should be increased and often exceed the theoretical extent of thin shells; transverse shear deformation must be taken into account accordingly. Consequently, the effect of transverse shear deformation on the shells must be also considered; the corresponding calculated models and analytical theories should be established, which have induced increasing attention from scholars.

Since Sophie Germain (1813) gained correct expression of bending of thin plate and Aaron (1874) and Love (1888) established theory of thin shells; the theory of plates and shells have been developed with the shells structure gradually application in engineering [3]. Based on Kirchhoff-Love's classical theory of thin shells [4], the comparatively perfect result of dynamic excitation was obtained within the low radio-wave band when $R / h \gg 1$. However, it may cause $30 \%$ or even more error by putting this theory into the deformation, stress, and frequency analysis of composite shells, because the ratio of their elastic modulus and shear modulus usually reach $25-40$. Therefore, the CST is not feasible for this case [5].

High-order shear deformation theory (HSDT) and FSDT are adopted to analyse the effect of transverse shearing deformation in the moderately thick plates and shells theory [6-8]. The HSDT can better satisfy the boundary conditions of upper and lower surface of shells, but the equation is more complex, and some of its components of internal forces have no clear physical significance. In contrary, although the FSDT cannot always satisfy the unstressed boundary conditions of upper and lower surface of shells, it can get a simpler equation and readily establish the boundary conditions, with clear physical significance of component internal forces. Thus, the FSDT has been commonly used in actual engineering. Furthermore, the transverse shear deformation with increased 


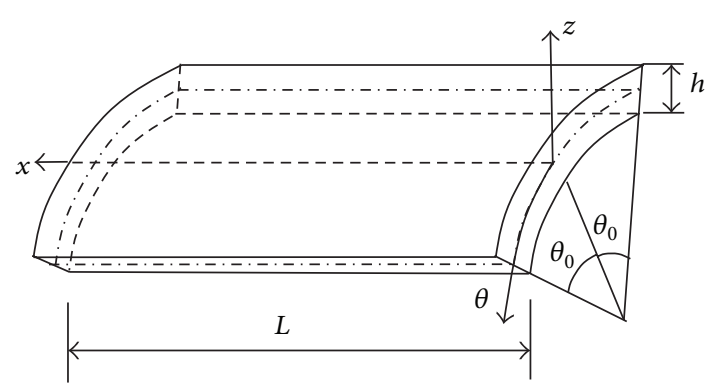

FIGURE 1: Coordinate system of cylindrical shells.

equation order of shells brings tremendous difficulties on solving the equation. Therefore, whether the new equation can be solved in transverse shear deformation is another tough problem during the research of bending problem of moderately thick shells $[9,10]$. By far, few analytical solutions of bending problem of moderately thick shells have been obtained based on the FSDT. This paper presents the basic equation of static forces calculation of cylindrical shells on the basis of the FSDT and derives the control equation of moderately thick cylindrical shells by introducing displacement potential functions. Furthermore, the subject of static analysis of moderately thick cylindrical ribbed shells is an interaction problem of edge beam and structure and with great complexity. Therefore, current research has been carried out mostly on thin cylindrical shells. The edge force at the longitudinal of shells has been obtained based on the derivation of control differential equation of moderately thick cylindrical shell. Meanwhile, the displacement of edge beam has been deduced under the action of edge force. In addition, the join condition of moderately thick cylindrical ribbed shells is established according to the continuity of displacement, and the displacement analytical solution of bending problems of moderately thick cylindrical ribbed shells has been obtained as well.

\section{The Governing Equation of Moderately Thick Cylindrical Shells Based on FSDT}

Take coordinate system of cylindrical shells as shown in Figure 1:

$$
\alpha_{1}=x, \quad \alpha_{2}=\theta .
$$

Then the corresponding Lamé coefficients are

$$
A_{1}=1, \quad A_{2}=1 .
$$

The radiuses of curvature are

$$
R_{1}=\infty, \quad R_{2}=R
$$

Hence, the geometrical equations can be written as

$$
\begin{gathered}
\varepsilon_{11}=\frac{\partial u_{1}}{\partial x}, \quad \varepsilon_{22}=\frac{\partial u_{2}}{R \partial x}+\frac{w}{R}, \\
w=\frac{\partial u_{1}}{R \partial \theta}+\frac{\partial u_{2}}{\partial x}, \quad \kappa_{1}=\frac{\partial \phi_{1}}{\partial x}, \quad \kappa_{2}=\frac{\partial \phi_{2}}{R \partial \theta},
\end{gathered}
$$

$$
\begin{gathered}
\tau=\tau_{12}+\tau_{21}=\frac{1}{R} \frac{\partial \phi_{1}}{\partial \theta}+\frac{\partial \phi_{2}}{\partial x} \\
\varepsilon_{13}=\phi_{1}+\frac{\partial w}{\partial x}, \quad \varepsilon_{23}=\phi_{2}+\frac{\partial w}{R \partial \theta} .
\end{gathered}
$$

And the constitutive equations are

$$
\begin{gathered}
T_{11}=K\left(\varepsilon_{11}+\nu \varepsilon_{22}\right), \quad T_{22}=K\left(\varepsilon_{22}+\nu \varepsilon_{11}\right), \\
T_{12}=T_{21}=K \frac{1-\nu}{2} \omega, \\
M_{11}=D\left(\kappa_{1}+\nu \kappa_{2}\right), \quad M_{22}=D\left(\kappa_{2}+\nu \kappa_{1}\right), \\
M_{12}=M_{21}=D \frac{1-\nu}{2} \tau, \\
T_{1 n}=\frac{G h}{k_{\tau}}\left(\phi_{1}+\frac{\partial w}{\partial x}\right), \quad T_{2 n}=\frac{G h}{k_{\tau}}\left(\phi_{2}+\frac{\partial w}{R \partial \theta}\right) .
\end{gathered}
$$

Also, the equilibrium equations can be written as

$$
\begin{gathered}
\frac{R \partial T_{11}}{\partial x}+\frac{\partial T_{21}}{\partial \theta}=-R q_{1} \\
\frac{\partial T_{22}}{\partial \theta}+\frac{R \partial T_{12}}{\partial x}+T_{2 n}=-R q_{2} \\
T_{22}-\frac{R \partial T_{1 n}}{\partial x}-\frac{\partial T_{2 n}}{\partial \theta}=R q_{n} \\
\frac{\partial M_{22}}{\partial \theta}+\frac{R \partial M_{12}}{\partial x}=R T_{2 n} \\
\frac{R \partial M_{11}}{\partial x}+\frac{\partial M_{21}}{\partial \theta}=R T_{1 n}
\end{gathered}
$$

where $J_{0}=h, J_{2}=h^{3} / 12, K=E h /\left(1-v^{2}\right), D=E h^{3} / 12(1-$ $\left.v^{2}\right), K$ is the tensile stiffness of the shell, and $D$ is the bending stiffness of the shell.

Substituting (2) into (3), the internal force and the bending moment, which are determined by $u_{1}, u_{2}, w, \phi_{1}$, and $\phi_{2}$, can be obtained. Then, substituting the internal force and the bending moment into $(4 \mathrm{a})-(4 \mathrm{e})$, the displacement-based differential equation set of moderately thick cylindrical shells can be obtained as

$$
\begin{aligned}
& \frac{\partial^{2} u_{1}}{\partial x^{2}}+\frac{1-v}{2} \frac{\partial^{2} u_{1}}{R^{2} \partial \theta^{2}}+\frac{1+v}{2} \frac{\partial^{2} u_{2}}{R \partial x \partial \theta}+\frac{v}{R} \frac{\partial w}{\partial x}=-\frac{q_{1}}{K}, \\
& \frac{\partial^{2} u_{2}}{R^{2} \partial \theta^{2}}+\frac{1-v}{2} \frac{\partial^{2} u_{2}}{\partial x^{2}}+\frac{1+v}{2} \frac{\partial^{2} u_{1}}{R \partial x \partial \theta}+\frac{1}{R^{2}} \frac{\partial w}{\partial \theta}=-\frac{q_{2}}{K}, \\
& \frac{1}{R}\left(\frac{\partial u_{2}}{R \partial \theta}+\frac{w}{R}+v \frac{\partial u_{1}}{\partial x}\right)-k\left(\nabla^{2} w+\frac{\partial \phi_{1}}{\partial x}+\frac{\partial \phi_{2}}{R \partial \theta}\right)=\frac{q_{n}}{K},
\end{aligned}
$$




$$
\frac{\partial^{2} \phi_{1}}{\partial x^{2}}+\frac{1-v}{2} \frac{\partial^{2} \phi_{1}}{R^{2} \partial \theta^{2}}+\frac{1+v}{2} \frac{\partial^{2} \phi_{2}}{R \partial x \partial \theta}-k \frac{12}{h^{2}}\left(\phi_{1}+\frac{\partial w}{\partial x}\right)=0,
$$

$\frac{\partial^{2} \phi_{2}}{R^{2} \partial \theta^{2}}+\frac{1-\nu}{2} \frac{\partial^{2} \phi_{2}}{\partial x^{2}}+\frac{1+\nu}{2} \frac{\partial^{2} \phi_{1}}{R \partial x \partial \theta}-k \frac{12}{h^{2}}\left(\phi_{2}+\frac{\partial w}{R \partial \theta}\right)=0$,

where $k=(1-v) / 2 k_{\tau}$.

Bending problems of moderately thick cylindrical shells are summarized as mathematical problems that solve the displacement-based differential equation (2) under certain boundary conditions. Equations (5a)-(5e) are the differential equations composed of five second-order differential equations, whose solution is quite difficult; therefore, we introduce the displacement functions $F_{1}(x, \theta), F_{2}(x, \theta)$, and $f(x, \theta)$, and then we have

$$
\begin{gathered}
u_{1}=-\frac{1-v}{2 R}\left(\nu \frac{\partial^{3}}{\partial x^{3}}-\frac{\partial^{3}}{R^{2} \partial x \partial \theta^{2}}\right) F_{1}, \\
u_{2}=-\frac{1-v}{2 R}\left[(2+v) \frac{\partial^{3}}{R \partial x^{2} \partial \theta}+\frac{\partial^{3}}{R^{3} \partial \theta^{3}}\right] F_{1}, \\
w=\frac{1-v}{2} \nabla^{2} \nabla^{2} F_{1} .
\end{gathered}
$$

In the equation $\nabla^{2}=\partial^{2} / \partial x^{2}+\partial^{2} / R^{2} \partial \theta^{2}$, (5a) and (5b) automatically satisfy themselves.

Meanwhile, we can get

$$
\begin{gathered}
\phi_{1}=\frac{\partial F_{2}}{\partial x}-\frac{\partial f}{R \partial \theta}, \\
\phi_{2}=\frac{\partial F_{2}}{R \partial \theta}+\frac{\partial f}{\partial x} .
\end{gathered}
$$

Substituting (7a) and (7b) into (5d) and (5e) leads to

$$
\begin{gathered}
\frac{\partial}{\partial x}\left(\nabla^{2} F_{2}-\frac{12 k}{h^{2}} F_{2}-\frac{12 k}{h^{2}} w\right) \\
-\frac{\partial}{R \partial \theta}\left(\frac{1-v}{2} \nabla^{2} f-\frac{12 k}{h^{2}} f\right)=0, \\
\frac{\partial}{R \partial \theta}\left(\nabla^{2} F_{2}-\frac{12 k}{h^{2}} F_{2}-\frac{12 k}{h^{2}} w\right) \\
+\frac{\partial}{\partial x}\left(\frac{1-v}{2} \nabla^{2} f-\frac{12 k}{h^{2}} f\right)=0 .
\end{gathered}
$$

By the Cauchy-Riemann conditions, it does not lose its generality [11]; we obtain the following equations by using (8a) and (8b):

$$
\begin{gathered}
\nabla^{2} F_{2}-\frac{12 k}{h^{2}} F_{2}-\frac{12 k}{h^{2}} w=0, \\
\frac{1-v}{2} \nabla^{2} f-\frac{12 k}{h^{2}} f=0 .
\end{gathered}
$$

Substituting (6c) into (9a), the equation that functions $F_{1}(x, \theta)$ and $F_{2}(x, \theta)$ should satisfy can be established as

$$
\nabla^{2} F_{2}-k_{0} F_{2}=k_{0} \frac{1-\nu}{2} \nabla^{4} F_{1}
$$

where $k_{0}=12 k / h^{2}$. From $(6 a)-(6 c)$ and $(9 a)-(9 b),(5 a)-(5 e)$ can be satisfied. Then, substituting (6a)-(6c) and (7a)-(7b) into $(5 \mathrm{c})$ leads to

$$
\frac{1}{R}\left[\frac{1-v}{2 R}\left(1-v^{2}\right) \frac{\partial^{4} F_{1}}{\partial x^{4}}\right]-k\left[\frac{1-v}{2} \nabla^{6} F_{1}+\nabla^{2} F_{2}\right]=\frac{q_{n}}{K} .
$$

Equations (10), (11), and (9b) are equivalent to (5a)-(5e). In order to make them a single equation, we have

$$
\begin{aligned}
& F_{1}=\left(\nabla^{2}-k_{0}\right) F, \\
& F_{2}=k_{0} \frac{1-v}{2} \nabla^{4} F .
\end{aligned}
$$

Then (10) automatically satisfies itself. In (12a) and (12b), $F(x, y)$ is another unknown function. By substituting (12a) and (12b) into (11), we obtain the equation that function $F(x, \theta)$ should satisfy; that is,

$$
\begin{gathered}
k \frac{1-v}{2} \nabla^{8} F-\frac{(1-v)\left(1-v^{2}\right)}{2 R^{2}} \frac{\partial^{4}}{\partial x^{4}} \nabla^{2} F \\
+\frac{(1-v)\left(1-v^{2}\right)}{2 R^{2}} k_{0} \frac{\partial^{4} F}{\partial x^{4}}=-\frac{q_{n}}{K}
\end{gathered}
$$

To make the data easily analyzed, we introduce the nondimensional coordinate $\xi=x / L$ and make $\alpha_{1}=R / L$, $\alpha_{2}=h / R$. Thus, the governing differential equations (13) and (9b) can be, respectively, replaced by the following forms:

$$
\begin{gathered}
k \frac{1-\nu}{2} \nabla^{8} \widetilde{F}-\frac{(1-\nu)\left(1-\nu^{2}\right) \alpha_{1}^{4}}{2 R^{6}} \frac{\partial^{4}}{\partial \xi^{4}} \nabla^{2} \widetilde{F} \\
+\frac{6 k(1-\nu)\left(1-\nu^{2}\right) \alpha_{1}^{4}}{R^{8} \alpha_{2}^{2}} \frac{\partial^{4} \widetilde{F}}{\partial \xi^{4}}=-\frac{q_{n}}{K}, \\
\frac{1-\nu}{2} \nabla^{2} \widetilde{f}-\frac{12 k}{R^{2} \alpha_{2}^{2}} \widetilde{f}=0,
\end{gathered}
$$

where

$$
\begin{gathered}
\nabla^{8}(:)=\frac{1}{R^{8}}\left(\alpha_{1}^{8} \frac{\partial^{8}}{\partial \xi^{8}}+4 \alpha_{1}{ }^{6} \frac{\partial^{8}}{\partial \xi^{6} \partial \theta^{2}}+6 \alpha_{1}^{4} \frac{\partial^{8}}{\partial \xi^{4} \partial \theta^{4}}\right. \\
\left.+4 \alpha_{1}^{2} \frac{\partial^{8}}{\partial \xi^{2} \partial \theta^{6}}+\frac{\partial^{8}}{\partial \theta^{8}}\right)(:) \\
\nabla^{2}(:)=\frac{1}{R^{2}}\left(\alpha_{1}{ }^{2} \frac{\partial^{2}}{\partial \xi^{2}}+\frac{\partial^{2}}{\partial \theta^{2}}\right)(:) .
\end{gathered}
$$

Equations (12a) and (12b) can be written as

$$
\begin{aligned}
& \widetilde{F}_{1}=\frac{1}{R^{2}}\left(\alpha_{1}{ }^{2} \frac{\partial^{2}}{\partial \xi^{2}}+\frac{\partial^{2}}{\partial \theta^{2}}-\frac{12 k}{\alpha_{2}^{2}}\right) \widetilde{F}, \\
& \widetilde{F}_{2}=\frac{6 k(1-\nu)}{R^{6} \alpha_{2}{ }^{2}}\left(\alpha_{1}{ }^{4} \frac{\partial^{4}}{\partial \xi^{4}}+2 \alpha_{1}{ }^{2} \frac{\partial^{4}}{\partial \xi^{2} \partial \theta^{2}}+\frac{\partial^{4}}{\partial \theta^{4}}\right) \widetilde{F} .
\end{aligned}
$$


The displacement equations can be obtained by (6a)-(6c), which are

$$
\begin{aligned}
& \widetilde{u}_{1}=-\frac{1-v}{2 R^{4}}\left(\nu \alpha_{1}{ }^{3} \frac{\partial^{3}}{\partial \xi^{3}}-\alpha_{1} \frac{\partial^{3}}{\partial \xi \partial \theta^{2}}\right) \widetilde{F}_{1}, \\
& \widetilde{u}_{2}=-\frac{1-v}{2 R^{4}}\left[(2+v) \alpha_{1}{ }^{2} \frac{\partial^{3}}{\partial \xi^{2} \partial \theta}+\frac{\partial^{3}}{\partial \theta^{3}}\right] \widetilde{F}_{1}, \\
& \widetilde{w}=\frac{1-\nu}{2 R^{4}}\left(\alpha_{1}{ }^{4} \frac{\partial^{4}}{\partial \xi^{4}}+2 \alpha_{1}{ }^{2} \frac{\partial^{4}}{\partial \xi^{2} \partial \theta^{2}}+\frac{\partial^{4}}{\partial \theta^{4}}\right) \widetilde{F}_{1} .
\end{aligned}
$$

And the angles of rotation can be expressed as

$$
\begin{aligned}
& \widetilde{\phi}_{1}=\frac{1}{R}\left(\alpha_{1} \frac{\partial \widetilde{F}_{2}}{\partial \xi}-\frac{\partial \widetilde{f}}{\partial \theta}\right), \\
& \widetilde{\phi}_{2}=\frac{1}{R}\left(\frac{\partial \widetilde{F}_{2}}{\partial \theta}+\alpha_{1} \frac{\partial \widetilde{f}}{\partial \xi}\right) .
\end{aligned}
$$

According to (2), the deformation can be obtained as

$$
\begin{gathered}
\widetilde{\varepsilon}_{11}=\frac{\alpha_{1}}{R} \frac{\partial \widetilde{u}_{1}}{\partial \xi}, \quad \widetilde{\varepsilon}_{22}=\frac{1}{R}\left(\frac{\partial \widetilde{u}_{2}}{\partial \theta}+\widetilde{w}\right), \\
\widetilde{\omega}=\frac{1}{R}\left(\frac{\partial \widetilde{u}_{1}}{\partial \theta}+\alpha_{1} \frac{\partial \widetilde{u}_{2}}{\partial \xi}\right), \\
\widetilde{\kappa}_{1}=\frac{\alpha_{1}}{R} \frac{\partial \widetilde{\phi}_{1}}{\partial \xi}, \quad \widetilde{\kappa}_{2}=\frac{1}{R} \frac{\partial \widetilde{\phi}_{2}}{\partial \theta}, \quad \tilde{\tau}=\frac{\alpha_{1}}{R} \frac{\partial \widetilde{\phi}_{2}}{\partial \xi}+\frac{1}{R^{2}} \frac{\partial \widetilde{\phi}_{1}}{\partial \theta}, \\
\widetilde{\varepsilon}_{13}=\left(\widetilde{\phi}_{1}+\frac{\alpha_{1}}{R} \frac{\partial \widetilde{w}}{\partial \xi}\right), \quad \widetilde{\varepsilon}_{23}=\widetilde{\phi}_{2}+\frac{1}{R^{2}} \frac{\partial \widetilde{w}}{\partial \theta} .
\end{gathered}
$$

According to (3), internal forces can be obtained as

$$
\begin{gathered}
\widetilde{T}_{11}=\frac{E R \alpha_{2}}{1-v^{2}}\left(\widetilde{\varepsilon}_{11}+v \widetilde{\varepsilon}_{22}\right), \\
\widetilde{T}_{22}=\frac{E R \alpha_{2}}{1-v^{2}}\left(\widetilde{\varepsilon}_{22}+v \widetilde{\varepsilon}_{11}\right), \\
\widetilde{M}_{11}=\frac{E R^{3} \alpha_{2}^{3}}{12\left(1-v^{2}\right)}\left(\widetilde{\kappa}_{1}+v \widetilde{\kappa}_{2}\right), \\
\widetilde{M}_{22}=\frac{E R^{3} \alpha_{2}^{3}}{12\left(1-v^{2}\right)}\left(\widetilde{\kappa}_{2}+v \widetilde{\kappa}_{1}\right), \\
\widetilde{M}_{12}=\widetilde{M}_{21}=\frac{E R^{3} \alpha_{2}^{3}}{24(1+v)} \widetilde{\tau}, \\
\widetilde{T}_{12}=\widetilde{T}_{21}=\frac{E R \alpha_{2}}{1-v^{2}} \frac{1-v}{2} \widetilde{\omega}_{T}, \\
\widetilde{T}_{13}=\frac{G R}{k_{\tau}} \alpha_{2} \widetilde{\varepsilon}_{13}, \quad \widetilde{T}_{23} \frac{G R}{k_{\tau}} \alpha_{2} \widetilde{\varepsilon}_{23} .
\end{gathered}
$$

By opening up the displacement function as trigonometric series in satisfying the boundary conditions, the control equation can be simplified to a solvable eighth-order ordinary differential equation and a second-order ordinary differential equation. After solving the solution of the control equation sets, displacement, angle, and internal force can be solved too, and then set ten undetermined coefficients that it contains by using boundary conditions or join conditions to solve the solution of moderately thick cylindrical shells.

\section{The Static Bending of Moderately Thick Cylindrical Shells That Considers the Effect of Edge Beam}

3.1. Displacement of Edge Beam under Edge Force. In order to study the coworking situation of shells and edge beam, the shells and edge beam are cut and split along the edge of the shells, this section will have the internal forces $T_{22}, T_{21}, T_{23}$, $M_{22}$, and $M_{21}$ that can balance with the external force and the corresponding deformations $u_{2}, u_{1}, w, \phi_{1}$, and $\phi_{2}$, as shown in Figure 2.

Now let us discuss the displacement state of bearing load of edge beams $T_{22}, T_{21}, T_{23}, M_{22}$ and $M_{21}$; the edge beam section adopts the coordinate system $(x, 1,2)$, among which coordinate $x$ locates in $y$-direction of edge beam, coordinate axes 1 and 2 stand for main axes of section moments of inertia, the point $O$ on the section shows the edge point on the edge beam equivalent to $r=R$ of shells, and, in addition, it has coordinates $e_{1}, e_{2}$, as shown in Figure 3.

In order to calculate the deformation state of edge beam, the edge beam bears the edge load as shown in Figure 4, and the rules of continuous change of a set of unit forces $\bar{p}_{1}, \bar{p}_{2}$, $\bar{z}_{x}, \bar{m}_{x}$, and $\bar{m}_{1}$ on $x$-axis are stipulated as follows:

$$
\begin{gathered}
\bar{p}_{1}=1 \cdot \sum_{n=1}^{\infty} \sin \left(\frac{n \pi x}{L}\right), \quad \bar{p}_{2}=1 \cdot \sum_{n=1}^{\infty} \sin \left(\frac{n \pi x}{L}\right), \\
\bar{z}_{x}=1 \cdot \sum_{n=1}^{\infty} \sin \left(\frac{n \pi x}{L}\right), \quad \bar{m}_{x}=1 \cdot \sum_{n=1}^{\infty} \sin \left(\frac{n \pi x}{L}\right), \\
\bar{m}_{1}=1 \cdot \sum_{n=1}^{\infty} \sin \left(\frac{n \pi x}{L}\right) .
\end{gathered}
$$

According to the displacement differential equation of given beam and the Saint-Venant torsional equation [12, 13], by (21), we have

$$
\begin{aligned}
\frac{d^{4} \bar{\delta}_{1}}{d x^{4}} & =\frac{1}{E I_{2}} \sum_{n=1}^{\infty} \sin \left(\frac{n \pi x}{L}\right) \\
\frac{d^{4} \bar{\delta}_{2}^{1}}{d x^{4}} & =\frac{1}{E I_{1}} \sum_{n=1}^{\infty} \sin \left(\frac{n \pi x}{L}\right) \\
\frac{d^{2} \bar{\delta}_{2}^{2}}{d x^{2}} & =\frac{1}{E I_{1}} \sum_{n=1}^{\infty} \sin \left(\frac{n \pi x}{L}\right) \\
\frac{d \bar{\delta}_{3}}{d x} & =\frac{1}{E F} \sum_{n=1}^{\infty} \sin \left(\frac{n \pi x}{L}\right)
\end{aligned}
$$



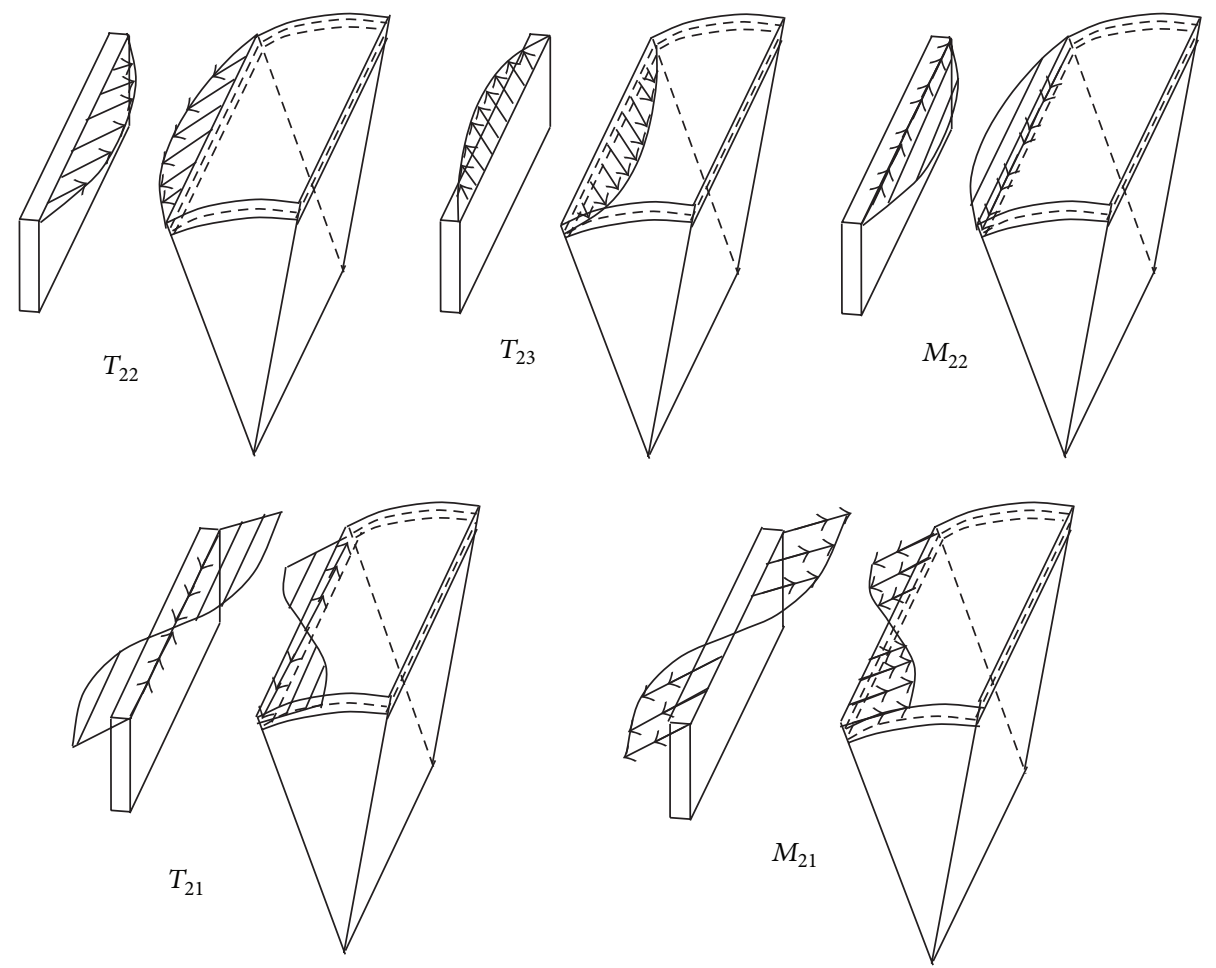

FIGURE 2: Force at the place where the shells link up with the edge beam.

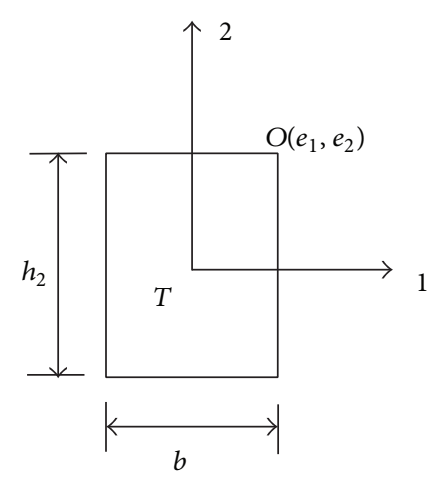

FIGURE 3: Coordinate graph of edge beam.
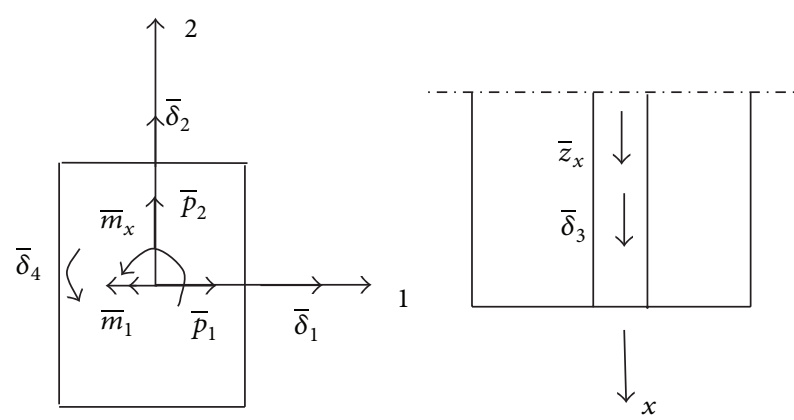

Figure 4: Rules of a set of displacement and stress on shear center $T$.

$$
\begin{gathered}
\frac{d^{2} \bar{\delta}_{4}}{d x^{2}}=-\frac{1}{G I_{t}} \sum_{n=1}^{\infty} \sin \left(\frac{n \pi x}{L}\right) \\
\bar{\delta}_{5}=\frac{d \bar{\delta}_{2}}{d x}=\frac{d \bar{\delta}_{2}{ }^{1}}{d x}+\frac{d \bar{\delta}_{2}^{2}}{d x} .
\end{gathered}
$$

The displacements $\bar{\delta}_{1}, \bar{\delta}_{2}, \bar{\delta}_{3}, \bar{\delta}_{4}$, and $\bar{\delta}_{5}$ are the ones of shear center $T$ corresponding to the directions of $\bar{p}_{1}, \bar{p}_{2}, \bar{z}_{x}$, $\bar{m}_{x}$, and $\bar{m}_{1}$.

Defining integral to (22) and making $l=L / n \pi$ lead to

$$
\begin{aligned}
& \bar{\delta}_{1}=\sum_{n=1}^{\infty} \frac{l^{4}}{E I_{2}} \sin \left(\frac{n \pi x}{L}\right), \\
& \bar{\delta}_{2}^{1}=\sum_{n=1}^{\infty} \frac{l^{4}}{E I_{1}} \sin \left(\frac{n \pi x}{L}\right), \\
& \bar{\delta}_{2}^{2}=-\sum_{n=1}^{\infty} \frac{l^{2}}{E I_{1}} \sin \left(\frac{n \pi x}{L}\right), \\
& \bar{\delta}_{3}=-\sum_{n=1}^{\infty} \frac{l}{E F} \cos \left(\frac{n \pi x}{L}\right), \\
& \bar{\delta}_{4}=\sum_{n=1}^{\infty} \frac{l^{2}}{G I_{t}} \sin \left(\frac{n \pi x}{L}\right) .
\end{aligned}
$$



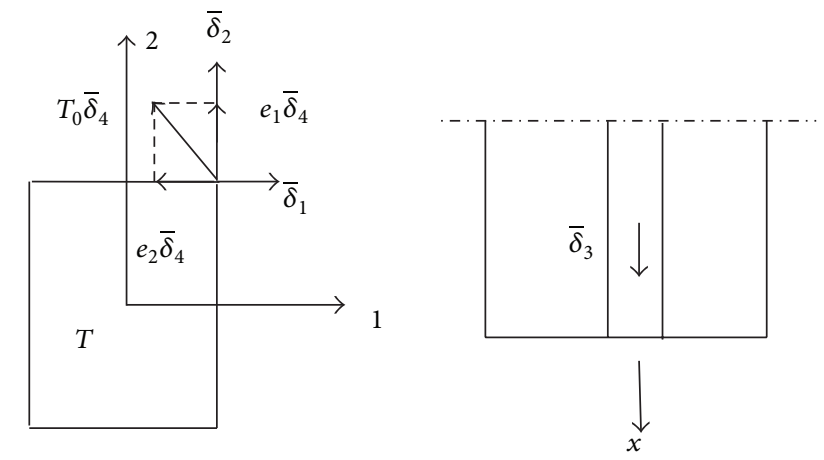

FIgURE 5: Displacement rules of point $O$.

The displacement on the point $O$ caused by the action of a set of force $\bar{p}_{1}=1, \bar{p}_{2}=1, \bar{z}_{x}=1, \bar{m}_{x}=1$, and $\bar{m}_{1}=1$ on point $T$ is listed in Table 1 .

In the table, the first column of the first row represents the displacement $\bar{\delta}_{1}$ of point $O$ in the direction of axis 1 caused by the action of force $\bar{p}_{1}=1$ on point $T$ along the axis 1; the second column of the first row stands for the displacement $\bar{\delta}_{1}$ of point $O$ in the direction of axis 1 caused by the action of force $\bar{p}_{2}=1$ on point $T$ along the axis 2 ; as axes 1 and 2 are orthogonal, $\bar{\delta}_{1}=0$; the third column of the first row represents the displacement $\bar{\delta}_{1}$ of point $O$ in the direction of axis 1 caused by the action of force $\bar{z}_{x}=1$ on point $T$ along the axis 1 ; as axes 1 and $x$ are orthogonal, $\bar{\delta}_{1}=0$; the fourth column of the first row stands for the displacement $\bar{\delta}_{1}$ of point $O$ in the direction of axis 1 caused by the action of moment of force $\bar{m}_{x}=1$ on point $T$ when bypassing the vertical axis of point $T$; the fifth column of the first row represents the displacement $\bar{\delta}_{1}$ of point $O$ in the direction of axis 1 caused by the action of moment of force $\bar{m}_{1}=1$ on point $T$ when rotating around axis 1 . The state of the remaining rows and columns can be obtained according to this method. The displacement rules of point $O$ of edge beam are as shown in Figure 5.

Generally, the edge force of shells often acts on the edge of beam, instead of acting on point $T$ directly; therefore, the edge force should be converted into a set of equivalent forces $\bar{p}_{1}, \bar{p}_{2}, \bar{z}_{x}, \bar{m}_{x}$, and $\bar{m}_{1}$ that act on point $T$. When taking $d x$ of beams during converting, the load $T_{21}=1 \cdot \sum_{n=1}^{\infty} \cos (n \pi x / L)$ and internal forces $M_{1}, Q_{2}, M_{2}, Q_{1}$, and $\bar{z}_{x}$ are acting on it.

From the equilibrium condition [14], we have

$$
\begin{gathered}
\frac{d M_{1}}{d x}=e_{2} \cdot \sum_{n=1}^{\infty} \cos \left(\frac{n \pi x}{L}\right), \quad \frac{d M_{2}}{d x}=e_{1} \cdot \sum_{n=1}^{\infty} \cos \left(\frac{n \pi x}{L}\right), \\
\frac{d \bar{z}_{x}}{d x}=-\sum_{n=1}^{\infty} \cos \left(\frac{n \pi x}{L}\right) .
\end{gathered}
$$

TABLE 1: Displacement on the point $O$ caused by unit force acting on point $T$.

\begin{tabular}{cccccc}
\hline & $\bar{p}_{1}=1$ & $\bar{p}_{2}=1$ & $\bar{z}_{x}=1$ & $\bar{m}_{x}=1$ & $\bar{m}_{1}=1$ \\
\hline $\bar{\delta}_{1}$ & $\frac{l^{4}}{E I_{2}}$ & 0 & 0 & $-\frac{l^{2} e_{2}}{G I_{t}}$ & 0 \\
$\bar{\delta}_{2}$ & 0 & $\frac{l^{4}}{E I_{1}}$ & 0 & $\frac{l^{2} e_{1}}{G I_{t}}$ & 0 \\
$\bar{\delta}_{3}$ & $\frac{l^{3} e_{1}}{E I_{2}}$ & $\frac{l^{3} e_{2}}{E I_{1}}$ & $-\frac{l}{E F}$ & 0 & $-\frac{l^{2}}{E I_{1}}$ \\
$\bar{\delta}_{4}$ & 0 & 0 & 0 & $\frac{l^{2}}{G I_{t}}$ & 0 \\
$\bar{\delta}_{5}$ & 0 & $\frac{l^{3}}{E I_{1}}$ & 0 & 0 & $-\frac{l}{E I_{1}}$ \\
\hline
\end{tabular}

TABLE 2: Equivalent load.

\begin{tabular}{lccccc}
\hline & $\bar{p}_{1}$ & $\bar{p}_{2}$ & $\bar{z}_{x}$ & $\bar{m}_{x}$ & $\bar{m}_{1}$ \\
\hline$p_{1}=1$ & 1 & 0 & 0 & $-e_{2}$ & 0 \\
$p_{2}=1$ & 0 & 1 & 0 & $e_{1}$ & 0 \\
$T_{21}=1$ & $-\frac{e_{1}}{l}$ & $-\frac{e_{2}}{l}$ & $-l$ & 0 & 0 \\
$M_{22}=1$ & 0 & 0 & 0 & 1 & 0 \\
$M_{21}=1$ & 0 & 0 & 0 & 0 & 1 \\
\hline
\end{tabular}
have

Defining differential and integral to (24), respectively, we

$$
\begin{aligned}
\frac{d^{2} M_{1}}{d x^{2}} & =-\sum_{n=1}^{\infty} \frac{e_{2}}{l} \sin \left(\frac{n \pi x}{L}\right), \\
\frac{d^{2} M_{2}}{d x^{2}} & =-\sum_{n=1}^{\infty} \frac{e_{1}}{l} \sin \left(\frac{n \pi x}{L}\right), \\
\bar{z}_{x} & =-\sum_{n=1}^{\infty} l \sin \left(\frac{n \pi x}{L}\right) .
\end{aligned}
$$

By comparing (25) with differential equations $d^{2} M_{1} / d x^{2}=p_{2}(x)$ and $d^{2} M_{2} / d x^{2}=p_{1}(x)$, it shows that the stress and displacement in the main axis 1 of moments of inertia and direction 2 caused by the action of $T_{21}=1 \cdot \sum_{n=1}^{\infty} \cos (n \pi x / L)$ can be replaced by equivalent axial force $\bar{z}_{x}^{\infty}=-\sum_{n=1}^{\infty} l \cdot \sin (n \pi x / L)$ and mathematical loads $-\sum_{n=1}^{\infty}\left(e_{1} / l\right) \sin (n \pi x / L)$ and $-\sum_{n=1}^{\infty}\left(e_{2} / l\right) \sin (n \pi x / L)$; therefore, the equivalent load can be calculated according to Table 2.

In Table 2, the first column of the first row represents the equivalent load $\bar{p}_{1}$ of the corresponding point $T$ on the axis 1 when point $O$ acting on $p_{1}=1$ along the axis 1 , whose value is 1 ; the second column of the first row stands for the equivalent load $\bar{p}_{2}$ of the corresponding point $T$ on the axis 2 when point $O$ acting on $p_{1}=1$ along the axis 1 ; as axes 1 and 2 are orthogonal, its value is 0 ; the third column of the first row represents the equivalent load $\bar{z}_{x}$ of the corresponding point $T$ on the $x$-axis when point $O$ acting on $p_{1}=1$ along the axis 1 ; as axis 1 and $x$-axis are orthogonal, its value is 0 ; the fourth column of the first row stands for the equivalent load $\bar{m}_{x}$ of the corresponding point $T$ through the vertical axis of point $T$ when point $O$ acting on $p_{1}=1$ along the axis 1 , 
TABle 3: Displacement of point $O$ caused by the action of unit force.

\begin{tabular}{cccccc}
\hline & $p_{1}=1$ & $p_{2}=1$ & $T_{21}=1$ & $M_{22}=1$ & $M_{21}=1$ \\
\hline$\delta_{1}$ & $\frac{l^{4}}{E I_{2}}+\frac{l^{2} e_{2}{ }^{2}}{G I_{t}}$ & $-\frac{l^{2} e_{1} e_{2}}{G I_{t}}$ & $-\frac{l^{3} e_{1}}{E I_{2}}$ & $-\frac{l^{2} e_{2}}{G I_{t}}$ & 0 \\
\hline$\delta_{2}$ & $-\frac{l^{2} e_{1} e_{2}}{G I_{t}}$ & $\frac{l^{4}}{E I_{1}}+\frac{l^{2} e_{1}{ }^{2}}{G I_{t}}$ & $-\frac{l^{3} e_{2}}{E I_{1}}$ & $\frac{l^{2} e_{1}}{G I_{t}}$ & $-\frac{l^{2}}{E I_{1}}$ \\
\hline$\delta_{3}$ & $\frac{l^{3} e_{1}}{E I_{2}}$ & $\frac{l^{3} e_{2}}{E I_{1}}$ & $-\frac{l^{2}}{E F}\left(\frac{F e_{1}{ }^{2}}{I_{2}}+\frac{F e_{2}{ }^{2}}{I_{1}}-1\right)$ & 0 & 0 \\
\hline$\delta_{4}$ & $-\frac{l^{2} e_{2}}{G I_{t}}$ & $\frac{l^{2} e_{1}}{G I_{t}}$ & 0 & $\frac{l^{2}}{G I_{t}}$ & 0 \\
\hline$\delta_{5}$ & 0 & $\frac{l^{3}}{E I_{1}}$ & $-\frac{l^{2} e_{2}}{E I_{1}}$ & 0 & $-\frac{l}{E I_{1}}$ \\
\hline
\end{tabular}

TABLE 4: Displacement of point $O$ caused by the action of unit force.

\begin{tabular}{|c|c|c|c|c|c|}
\hline & $p_{1}=1$ & $p_{2}=1$ & $T_{21}=1$ & $M_{22}=1$ & $M_{21}=1$ \\
\hline \multirow{4}{*}{$\delta_{1}$} & 12 & & & & \multirow{4}{*}{0} \\
\hline & $\overline{E \alpha_{3}\left(\alpha_{4} n \pi\right)^{4}}$ & -3 & -6 & -3 & \\
\hline & $+\frac{3}{2}$ & $\overline{4 G \alpha_{3}{ }^{2}\left(\alpha_{4} n \pi\right)^{2}}$ & $\overline{E \alpha_{3}\left(\alpha_{4} n \pi\right)^{3}}$ & $\overline{2 G \alpha_{3}{ }^{2}\left(\alpha_{4} n \pi\right)^{2} b}$ & \\
\hline & $4 G \alpha_{3}\left(\alpha_{4} n \pi\right)^{2}$ & & & & \\
\hline \multirow{4}{*}{$\delta_{2}$} & & 12 & \multirow{4}{*}{$\frac{-6}{E \alpha_{3}^{2}\left(\alpha_{4} n \pi\right)^{3}}$} & & \multirow{4}{*}{$\frac{-12}{E \alpha_{3}{ }^{3}\left(\alpha_{4} n \pi\right)^{2} b^{2}}$} \\
\hline & -3 & $\overline{E \alpha_{3}{ }^{3}\left(\alpha_{4} n \pi\right)^{4}}$ & & 3 & \\
\hline & $\overline{4 G \alpha_{3}^{2}\left(\alpha_{4} n \pi\right)^{2}}$ & $+\frac{3}{3}$ & & $\overline{2 G \alpha_{3}{ }^{3}\left(\alpha_{4} n \pi\right)^{2} b}$ & \\
\hline & & $4 G \alpha_{3}{ }^{3}\left(\alpha_{4} n \pi\right)^{2}$ & & & \\
\hline \multirow{2}{*}{$\delta_{3}$} & 6 & 6 & -5 & \multirow{2}{*}{0} & \multirow{2}{*}{0} \\
\hline & $\overline{E \alpha_{3}\left(\alpha_{4} n \pi\right)^{3}}$ & $\overline{E \alpha_{3}{ }^{2}\left(\alpha_{4} n \pi\right)^{3}}$ & $\overline{E \alpha_{3}\left(\alpha_{4} n \pi\right)^{2}}$ & & \\
\hline \multirow{2}{*}{$\delta_{4}$} & -3 & 3 & \multirow{2}{*}{0} & 3 & \multirow{2}{*}{0} \\
\hline & $\overline{2 G \alpha_{3}{ }^{2}\left(\alpha_{4} n \pi\right)^{2} b}$ & $\overline{2 G \alpha_{3}{ }^{3}\left(\alpha_{4} n \pi\right)^{2} b}$ & & $\overline{G \alpha_{3}{ }^{3}\left(\alpha_{4} n \pi\right)^{2} b^{2}}$ & \\
\hline \multirow{2}{*}{$\delta_{5}$} & 0 & 12 & -6 & \multirow{2}{*}{0} & -12 \\
\hline & & $\overline{E \alpha_{3}{ }^{3}\left(\alpha_{4} n \pi\right)^{3} b}$ & $\overline{E \alpha_{3}{ }^{2}\left(\alpha_{4} n \pi\right)^{2} b}$ & & $\overline{E \alpha_{3}{ }^{3} \alpha_{4} n \pi b}$ \\
\hline
\end{tabular}

whose value is $-e_{2}$; as its direction is opposite to the specified one, as shown in Figure 3, the negative sign is taken; the fifth column of the first row represents the equivalent load $\bar{m}_{1}$ of the corresponding point $T$ through the axis 1 when point $O$ acting on $p_{1}=1$ along the axis 1 , whose value is 1 .

By using the combination of the displacement of point $O$ caused by the action of $\bar{p}_{1}=1, \bar{p}_{2}=1, \bar{z}_{x}=1, \bar{m}_{x}=1$, and $\bar{m}_{1}=1$ of point $T$ shown in Table 1 and the equivalent load of point $T$ caused by the action of the edge force of point $O$ as shown in Table 2, displacements $\delta_{1}, \delta_{2}, \delta_{3}, \delta_{4}$, and $\delta_{5}$ of point $O$ on the cross section of edge beam caused by the action of unit forces $p_{1}=1, p_{2}=1, T_{21}=1, M_{22}=1$, and $M_{21}=1$ on the edge of point $O$ can be calculated according to Table 3 .

For the convenience of data analysis, record $\alpha_{3}=h_{2} / b$, $\alpha_{4}=b / L$, and rewrite all values in Table 3 as the form of $\alpha_{3}$ and $\alpha_{4}$, as shown in Table 4 .

3.2. Solution Method. Recently, open cylindrical shells, especially the reinforced concrete open cylindrical shells which are usually used to be large-span roofs, have been applied successfully in many projects. The curvy edges of cylindrical shells are braced by the reinforced concrete circular-arc thin plate or thin arch circle, which can be regarded as simply supported edges with large stiffness. Thus, we can introduce

$$
\widetilde{F}=\sum_{n=1}^{\infty} \widetilde{F}_{n}(\theta) \sin (n \pi \xi), \quad \tilde{f}=\sum_{n=1}^{\infty} \widetilde{f}_{n}(\theta) \cos (n \pi \xi),
$$

where $\xi=0$ and $\xi=1$ are the boundary conditions of simply supported edges.

Substituting (26) into (14a), we have

$$
\frac{d^{8} \widetilde{F}_{n}}{d \theta^{8}}+B_{1} \frac{d^{6} \widetilde{F}_{n}}{d \theta^{6}}+B_{2} \frac{d^{4} \widetilde{F}_{n}}{d \theta^{4}}+B_{3} \widetilde{F}_{n}^{\prime \prime}+B_{4} \widetilde{F}_{n}=-\frac{2 R^{8} q_{n}}{K k(1-\nu)} .
$$

Then, substituting (26) into (14b), we have

$$
\left[-\frac{1-v}{2}\left(\alpha_{1} n \pi\right)^{2}-\frac{12 k}{\alpha_{2}^{2}}\right] \tilde{f}_{n}+\frac{1-v}{2} \frac{d^{2} \widetilde{f}_{n}}{d \theta^{2}}=0,
$$


where

$$
\begin{aligned}
& B_{1}=-4\left(\alpha_{1} n \pi\right)^{2}, \\
& B_{2}=6\left(\alpha_{1} n \pi\right)^{4}, \\
& B_{3}=-4\left(\alpha_{1} n \pi\right)^{6}-\frac{1-v^{2}}{k}\left(\alpha_{1} n \pi\right)^{4}, \\
& B_{4}=\left(\alpha_{1} n \pi\right)^{8}+\frac{1-v^{2}}{k}\left(\alpha_{1} n \pi\right)^{6}+\frac{12\left(1-v^{2}\right)}{\alpha_{2}{ }^{2}}\left(\alpha_{1} n \pi\right)^{4} .
\end{aligned}
$$

The particular solution of $(27 a)$ is

$$
\widetilde{F}_{n}^{p}=-\frac{2 R^{8} q_{n}}{K k(1-v) B_{4}}
$$

To obtain the solution of (27a) and (27b), substituting $\widetilde{F}_{n}^{h}=e^{\lambda_{m} \theta}$ and $\widetilde{f}_{n}=e^{\lambda_{m} \theta}$ into (27a) and (27b), we have

$$
\begin{array}{r}
\lambda_{m}{ }^{8}+B_{1} \lambda_{m}{ }^{6}+B_{2} \lambda_{m}{ }^{4}+B_{3} \lambda_{m}{ }^{2}+B_{4}=0, \\
(m=1,2,3, \ldots, 8), \\
\lambda_{m}{ }^{2}=\left(\alpha_{1} n \pi\right)^{2}+\frac{24 k}{(1-v) \alpha_{2}^{2}} \quad(m=9,10) .
\end{array}
$$

Equation (30a) can be solved using MATLAB, which is composed of 8 complex roots

$$
\begin{array}{ll}
\lambda_{1,2}=d_{1} \pm d_{2} i, & \lambda_{3,4}=-d_{1} \pm d_{2} i, \\
\lambda_{5,6}=d_{3} \pm d_{4} i, & \lambda_{7,8}=-d_{3} \pm d_{4} i .
\end{array}
$$

The homogeneous solution of (27a) is

$$
\begin{aligned}
\widetilde{F}_{n}{ }^{h}= & e^{d_{1} \theta}\left[C_{1} \cdot \cos \left(d_{2} \theta\right)+C_{2} \cdot \sin \left(d_{2} \theta\right)\right] \\
& +e^{-d_{1} \theta}\left[C_{3} \cdot \cos \left(d_{2} \theta\right)+C_{4} \cdot \sin \left(d_{2} \theta\right)\right] \\
& +e^{d_{3} \theta}\left[C_{5} \cdot \cos \left(d_{4} \theta\right)+C_{6} \cdot \sin \left(d_{4} \theta\right)\right] \\
& +e^{-d_{3} \theta}\left[C_{7} \cdot \cos \left(d_{4} \theta\right)+C_{8} \cdot \sin \left(d_{4} \theta\right)\right]
\end{aligned}
$$

The general solution of $(27 a)$ is

$$
\widetilde{F}_{n}=\widetilde{F}_{n}^{h}+\widetilde{F}_{n}^{p}
$$

And the solution of (27b) is

$$
\tilde{f}_{n}=C_{9} e^{\lambda_{9} \theta}+C_{10} e^{\lambda_{10} \theta},
$$

where $C_{1} \sim C_{10}$ are undetermined coefficients, which can be determined by boundary conditions or join conditions.

To satisfy boundary conditions or join conditions, displacement components, stress components, and bending moment components are expanded according to trigonometric series.
According to (16a) and (16b), the expansions of displacement functions can be expressed as

$$
\begin{aligned}
\widetilde{F}_{1} & =\sum_{n=1}^{\infty} \frac{1}{R^{2}}\left[\frac{d^{2} \widetilde{F}_{n}}{d \theta^{2}}-\left(\frac{12 k}{\alpha_{2}{ }^{2}}+\left(\alpha_{1} n \pi\right)^{2}\right) \widetilde{F}_{n}\right] \sin (n \pi \xi) \\
& =\sum_{n=1}^{\infty} \widetilde{F}_{1 n}(\theta) \sin (n \pi \xi),
\end{aligned}
$$

$$
\begin{array}{r}
\widetilde{F}_{2}=\sum_{n=1}^{\infty} \frac{6 k(1-\nu)}{R^{6} \alpha_{2}{ }^{2}}[ \\
\left(\alpha_{1} n \pi\right)^{4} \widetilde{F}_{n}-2\left(\alpha_{1} n \pi\right)^{2} \\
\left.\cdot \frac{d^{2} \widetilde{F}_{n}}{d \theta^{2}}+\frac{d^{4} \widetilde{F}_{n}}{d \theta^{4}}\right] \sin (n \pi \xi)
\end{array}
$$

$$
=\sum_{n=1}^{\infty} \widetilde{F}_{2 n}(\theta) \sin (n \pi \xi) .
$$

According to (17a)-(17c), the expansions of displacement components can be expressed as

$$
\begin{aligned}
\tilde{u}_{1} & =\sum_{n=1}^{\infty} \frac{1-\nu}{2 R^{4}}\left[\nu\left(\alpha_{1} n \pi\right)^{3} \widetilde{F}_{1 n}+\alpha_{1} n \pi \frac{d^{2} \widetilde{F}_{1 n}}{d \theta^{2}}\right] \cos (n \pi \xi) \\
& =\sum_{n=1}^{\infty} \widetilde{u}_{1 n} \cos (n \pi \xi),
\end{aligned}
$$

$$
\begin{aligned}
\widetilde{u}_{2} & =\sum_{n=1}^{\infty} \frac{1-\nu}{2 R^{4}}\left[(2+\nu)\left(\alpha_{1} n \pi\right)^{2} \frac{d \widetilde{F}_{1 n}}{d \theta}-\frac{d^{3} \widetilde{F}_{1 n}}{d \theta^{3}}\right] \sin (n \pi \xi) \\
& =\sum_{n=1}^{\infty} \widetilde{u}_{2 n} \sin (n \pi \xi)
\end{aligned}
$$$$
\widetilde{w}=\sum_{n=1}^{\infty} \frac{1-\nu}{2 R^{4}}\left[\left(\alpha_{1} n \pi\right)^{4} \widetilde{F}_{1 n}-2\left(\alpha_{1} n \pi\right)^{2}\right.
$$$$
\left.\cdot \frac{d^{2} \widetilde{F}_{1 n}}{d \theta^{2}}+\frac{d^{4} \widetilde{F}_{1 n}}{d \theta^{4}}\right] \sin (n \pi \xi)
$$

$$
=\sum_{n=1}^{\infty} \widetilde{w}_{n} \sin (n \pi \xi) .
$$

According to (18a) and (18b), the expansions of angles of rotation can be expressed as

$\widetilde{\phi}_{1}=\sum_{n=1}^{\infty} \frac{1}{R}\left[\left(\alpha_{1} n \pi\right) \widetilde{F}_{2 n}-\frac{d \tilde{f}}{d \theta}\right] \cos (n \pi \xi)=\sum_{n=1}^{\infty} \widetilde{u}_{4 n} \cos (n \pi \xi)$,

$\widetilde{\phi}_{2}=\sum_{n=1}^{\infty} \frac{1}{R}\left[\frac{d \widetilde{F}_{2 n}}{d \theta}-\alpha_{1} n \pi \widetilde{f}_{n}\right] \sin (n \pi \xi)=\sum_{n=1}^{\infty} \widetilde{u}_{5 n} \sin (n \pi \xi)$. 
According to (19), the expansions of strain components can be expressed as

$$
\begin{aligned}
& \widetilde{\varepsilon}_{11}=\sum_{n=1}^{\infty}-\frac{\alpha_{1} n \pi}{R} \widetilde{u}_{1 n} \sin (n \pi \xi)=\sum_{n=1}^{\infty} \widetilde{\varepsilon}_{1 n} \sin (n \pi \xi), \\
& \widetilde{\varepsilon}_{22}= \sum_{n=1}^{\infty} \frac{1}{R}\left(\frac{d \widetilde{u}_{2 n}}{d \theta}+\widetilde{w}_{n}\right) \sin (n \pi \xi)=\sum_{n=1}^{\infty} \widetilde{\varepsilon}_{2 n} \sin (n \pi \xi), \\
& \widetilde{\omega}=\sum_{n=1}^{\infty} \frac{1}{R}\left(\frac{d \widetilde{u}_{1 n}}{d \theta}+\alpha_{1} n \pi \widetilde{u}_{2}\right) \cos (n \pi \xi)=\sum_{n=1}^{\infty} \widetilde{\omega}_{n} \cos (n \pi \xi), \\
& \widetilde{\kappa}_{1}= \sum_{n=1}^{\infty}-\frac{\alpha_{1} n \pi}{R} \widetilde{u}_{4 n} \sin (n \pi \xi)=\sum_{n=1}^{\infty} \widetilde{\kappa}_{1 n} \sin (n \pi \xi), \\
& \widetilde{\kappa}_{2}= \sum_{n=1}^{\infty} \frac{1}{R} \frac{d \widetilde{u}_{5 n}}{d \theta} \sin (n \pi \xi)=\sum_{n=1}^{\infty} \widetilde{\kappa}_{2 n} \sin (n \pi \xi), \\
& \widetilde{\tau}_{1}= \sum_{n=1}^{\infty} \frac{\alpha_{1} n \pi}{R} \widetilde{u}_{5 n} \cos (n \pi \xi)=\sum_{n=1}^{\infty} \widetilde{\tau}_{1 n} \cos (n \pi \xi), \\
& \widetilde{\tau}_{2}= \sum_{n=1}^{\infty} \frac{1}{R} \frac{d \widetilde{u}_{4 n}}{d \theta} \cos (n \pi \xi)=\sum_{n=1}^{\infty} \widetilde{\tau}_{2 n} \cos (n \pi \xi), \\
& \widetilde{\varepsilon}_{23}= \sum_{n=1}^{\infty}\left(\widetilde{u}_{5 n}+\frac{1}{R} \frac{d \widetilde{u}_{3 n}}{d \theta}\right) \sin (n \pi \xi)=\sum_{n=1}^{\infty} \widetilde{\varepsilon}_{23 n} \sin (n \pi \xi) . \\
&\left.\widetilde{u}_{4 n}+\frac{\alpha_{1} n \pi}{R} \widetilde{u}_{3 n}\right) \cos (n \pi \xi)=\sum_{n=1}^{\infty} \widetilde{\varepsilon}_{13 n} \cos (n \pi \xi),
\end{aligned}
$$

According to (20), the expansions of internal force and bending moment components can be expressed as

$$
\begin{aligned}
& \widetilde{T}_{11}= \frac{E R \alpha_{2}}{1-v^{2}} \sum_{n=1}^{\infty}\left(\widetilde{\varepsilon}_{1 n}+v \widetilde{\varepsilon}_{2 n}\right) \sin (n \pi \xi)=\sum_{n=1}^{\infty} \widetilde{T}_{1 n} \sin (n \pi \xi) \\
& \widetilde{T}_{22}=\frac{E R \alpha_{2}}{1-v^{2}} \sum_{n=1}^{\infty}\left(\widetilde{\varepsilon}_{2 n}+v \widetilde{\varepsilon}_{1 n}\right) \sin (n \pi \xi)=\sum_{n=1}^{\infty} \widetilde{T}_{2 n} \sin (n \pi \xi) \\
& \widetilde{M}_{11}=\frac{E R^{3} \alpha_{2}^{3}}{12\left(1-v^{2}\right)} \sum_{n=1}^{\infty}\left(\widetilde{\kappa}_{1 n}+v \widetilde{\kappa}_{2 n}\right) \sin (n \pi \xi) \\
&=\sum_{n=1}^{\infty} \widetilde{M}_{1 n} \sin (n \pi \xi), \\
& \widetilde{M}_{22}=\frac{E R^{3} \alpha_{2}{ }^{3}}{12\left(1-v^{2}\right)} \sum_{n=1}^{\infty}\left(\widetilde{\kappa}_{2 n}+v \widetilde{\kappa}_{1 n}\right) \sin (n \pi \xi) \\
&=\sum_{n=1}^{\infty} \widetilde{M}_{2 n} \sin (n \pi \xi), \\
& \widetilde{T}_{12}=\widetilde{T}_{21}=\frac{E R \alpha_{2} \sum_{2}^{\infty} \widetilde{\omega}_{n} \cos (n \pi \xi)=\sum_{n=1}^{\infty} \widetilde{T}_{12 n} \cos (n \pi \xi)}{2(1+v){ }_{n=1}}
\end{aligned}
$$

$$
\begin{aligned}
\widetilde{M}_{12} & =\widetilde{M}_{21}=\frac{E R^{3} \alpha_{2}^{3}}{24(1+v)} \sum_{n=1}^{\infty}\left(\widetilde{\tau}_{1 n}+v \widetilde{\tau}_{2 n}\right) \cos (n \pi \xi) \\
& =\sum_{n=1}^{\infty} \widetilde{M}_{12 n} \cos (n \pi \xi), \\
\widetilde{T}_{13} & =\frac{G R}{k_{\tau}} \alpha_{2} \sum_{n=1}^{\infty} \widetilde{\varepsilon}_{13 n} \cos (n \pi \xi)=\sum_{n=1}^{\infty} \widetilde{T}_{13 n} \cos (n \pi \xi) \\
\widetilde{T}_{23} & =\frac{G R}{k_{\tau}} \alpha_{2} \sum_{n=1}^{\infty} \widetilde{\varepsilon}_{23 n} \sin (n \pi \xi)=\sum_{n=1}^{\infty} \widetilde{T}_{23 n} \sin (n \pi \xi) .
\end{aligned}
$$

3.3. Join Conditions. There are five edge forces at the place where the shells is connected with the edge beam, namely, $\widetilde{T}_{21}, \widetilde{T}_{22}, \widetilde{T}_{23}, \widetilde{M}_{22}$, and $\widetilde{M}_{21}$. By opening them up with trigonometric series, we have

$$
\begin{gathered}
\widetilde{T}_{21}=\sum_{n=1}^{\infty} \widetilde{T}_{12 n} \cos (n \pi \xi), \quad \widetilde{T}_{22}=\sum_{n=1}^{\infty} \widetilde{T}_{2 n} \sin (n \pi \xi), \\
\widetilde{T}_{23}=\sum_{n=1}^{\infty} \widetilde{T}_{23 n} \sin (n \pi \xi), \quad \widetilde{M}_{21}=\sum_{n=1}^{\infty} \widetilde{M}_{12 n} \cos (n \pi \xi), \\
\widetilde{M}_{22}=\sum_{n=1}^{\infty} \widetilde{M}_{2 n} \sin (n \pi \xi),
\end{gathered}
$$

where $\widetilde{T}_{21}, \widetilde{T}_{22}, \widetilde{T}_{23}, \widetilde{M}_{22}$, and $\widetilde{M}_{21}$ are functions of $\theta$ and contain ten undetermined coefficients; then the edge forces are as follows:

on the edge I:

$$
\begin{aligned}
& \left.\widetilde{T}_{22}\right|_{\theta=-\theta_{0}} \\
& =\left.\sum_{n=1}^{\infty} \widetilde{T}_{2 n}\right|_{\theta=-\theta_{0}} \sin (n \pi \xi)=\sum_{n=1}^{\infty} r_{1 n} \sin (n \pi \xi)=r_{1}, \\
& \left.\widetilde{T}_{23}\right|_{\theta=-\theta_{0}} \\
& =\left.\sum_{n=1}^{\infty} \widetilde{T}_{23 n}\right|_{\theta=-\theta_{0}} \sin (n \pi \xi)=\sum_{n=1}^{\infty} r_{2 n} \sin (n \pi \xi)=r_{2}, \\
& \left.\left.\widetilde{T}_{21}\right|_{\theta=-\theta_{0}}{ }^{\infty} \widetilde{T}_{n=1}\right|_{\theta=-\theta_{0}} \cos (n \pi \xi)=\sum_{n=1}^{\infty} r_{3 n} \sin (n \pi \xi)=r_{3}, \\
& \left.\left.\widetilde{M}_{22}\right|_{\theta=-\theta_{0}} \widetilde{M}_{2 n}\right|_{\theta=-\theta_{0}} \sin (n \pi \xi)=\sum_{n=1}^{\infty} r_{4 n} \sin (n \pi \xi)=r_{4}, \\
& =\sum_{n=1}^{\infty}
\end{aligned}
$$




$$
\begin{aligned}
& \left.\widetilde{M}_{21}\right|_{\theta=-\theta_{0}} \\
& =\left.\sum_{n=1}^{\infty} \widetilde{M}_{12 n}\right|_{\theta=-\theta_{0}} \sin (n \pi \xi)=\sum_{n=1}^{\infty} r_{9 n} \sin (n \pi \xi)=r_{9} ;
\end{aligned}
$$

on the edge II:

$$
\begin{aligned}
& \left.\widetilde{T}_{22}\right|_{\theta=\theta_{0}} \\
& =\left.\sum_{n=1}^{\infty} \widetilde{T}_{2 n}\right|_{\theta=\theta_{0}} \sin (n \pi \xi)=\sum_{n=1}^{\infty} r_{5 n} \sin (n \pi \xi)=r_{5}, \\
& \left.\widetilde{T}_{23}\right|_{\theta=\theta_{0}} \\
& =\left.\sum_{n=1}^{\infty} \widetilde{T}_{23 n}\right|_{\theta=\theta_{0}} \sin (n \pi \xi)=\sum_{n=1}^{\infty} r_{6 n} \sin (n \pi \xi)=r_{6}, \\
& \left.\widetilde{T}_{21}\right|_{\theta=\theta_{0}} \\
& =\left.\sum_{n=1}^{\infty} \widetilde{T}_{12 n}\right|_{\theta=\theta_{0}} \cos (n \pi \xi)=\sum_{n=1}^{\infty} r_{7 n} \sin (n \pi \xi)=r_{7}, \\
& \left.\widetilde{M}_{22}\right|_{\theta=\theta_{0}} \\
& =\left.\sum_{n=1}^{\infty} \widetilde{M}_{2 n}\right|_{\theta=\theta_{0}} \sin (n \pi \xi)=\sum_{n=1}^{\infty} r_{8 n} \sin (n \pi \xi)=r_{8}, \\
& \left.\widetilde{M}_{21}\right|_{\theta=\theta_{0}} \\
& =\left.\sum_{n=1}^{\infty} \widetilde{M}_{12 n}\right|_{\theta=\theta_{0}} \sin (n \pi \xi)=\sum_{n=1}^{\infty} r_{10 n} \sin (n \pi \xi)=r_{10} .
\end{aligned}
$$

The edge forces are as shown in Figure 6.

In order to use the results in Table 4, we decompose the edge forces $r_{1} \sim r_{10}$ along axes 1 and 2 .

\section{Edge I}

Decompose $r_{1}, r_{2}$ along the axis 1:

$$
r_{1} \cos \theta-r_{2} \sin \theta=\sum_{n=1}^{\infty}\left(r_{1 n} \cos \theta-r_{2 n} \sin \theta\right) \sin (n \pi \xi)
$$

Decompose $r_{1}, r_{2}$ along the axis 2:

$$
r_{1} \sin \theta+r_{2} \cos \theta=\sum_{n=1}^{\infty}\left(r_{1 n} \sin \theta+r_{2 n} \cos \theta\right) \sin (n \pi \xi) \text {. }
$$

According to Table 4, the displacement generated by the edge beam I can be denoted by

$$
\begin{gathered}
\delta_{1}{ }^{\mathrm{I}}=\sum_{n=1}^{\infty} \delta_{1 n}{ }^{\mathrm{I}} \sin (n \pi \xi), \quad \delta_{2}{ }^{\mathrm{I}}=\sum_{n=1}^{\infty} \delta_{2 n}{ }^{\mathrm{I}} \sin (n \pi \xi), \\
\delta_{3}{ }^{\mathrm{I}}=\sum_{n=1}^{\infty} \delta_{3 n}{ }^{\mathrm{I}} \cos (n \pi \xi), \quad \delta_{4}{ }^{\mathrm{I}}=\sum_{n=1}^{\infty} \delta_{4 n}{ }^{\mathrm{I}} \sin (n \pi \xi), \\
\delta_{5}{ }^{\mathrm{I}}=\sum_{n=1}^{\infty} \delta_{5 n}{ }^{\mathrm{I}} \sin (n \pi \xi),
\end{gathered}
$$

where

$$
\begin{aligned}
& b_{0}=\frac{12}{E \alpha_{3}\left(\alpha_{4} n \pi\right)^{4}}+\frac{3}{4 G \alpha_{3}\left(\alpha_{4} n \pi\right)^{2}}, \\
& b_{1}=-\frac{3}{4 G \alpha_{3}^{2}\left(\alpha_{4} n \pi\right)^{2}}, \\
& b_{2}=-\frac{6}{E \alpha_{3}\left(\alpha_{4} n \pi\right)^{3}}, \quad b_{3}=-\frac{3}{2 G \alpha_{3}^{2}\left(\alpha_{4} n \pi\right)^{2} b} \text {, } \\
& c_{1}=\frac{12}{E \alpha_{3}{ }^{3}\left(\alpha_{4} n \pi\right)^{4}}+\frac{3}{4 G \alpha_{3}{ }^{3}\left(\alpha_{4} n \pi\right)^{2}}, \\
& c_{2}=-\frac{6}{E \alpha_{3}^{2}\left(\alpha_{4} n \pi\right)^{3}}, \\
& c_{3}=\frac{3}{2 G \alpha_{3}{ }^{3}\left(\alpha_{4} n \pi\right)^{2} b}, \quad c_{4}=\frac{12}{E \alpha_{3}{ }^{3}\left(\alpha_{4} n \pi\right)^{2} b^{2}}, \\
& d_{0}=-\frac{5}{E \alpha_{3}\left(\alpha_{4} n \pi\right)^{2}}, \quad f_{0}=\frac{3}{G \alpha_{3}^{3}\left(\alpha_{4} n \pi\right)^{2} b^{2}} \\
& g_{0}=\frac{12}{E \alpha_{3}{ }^{3}\left(\alpha_{4} n \pi\right)^{3} b}, \quad g_{1}=-\frac{6}{E \alpha_{3}{ }^{2}\left(\alpha_{4} n \pi\right)^{2} b} \text {, } \\
& g_{2}=-\frac{12}{E \alpha_{3}{ }^{3} \alpha_{4} n \pi b} \text {. }
\end{aligned}
$$

Thus, the subdisplacement and angle of rotation of edge beam $\mathrm{I}$ in $u, v$, and $w$ directions are

$$
\begin{aligned}
& \widetilde{u}_{1}{ }^{\mathrm{I}}=\delta_{3}{ }^{\mathrm{I}}=\sum_{n=1}^{\infty} \delta_{3 n}{ }^{\mathrm{I}} \cos (n \pi \xi), \\
& \widetilde{u}_{2}{ }^{\mathrm{I}}=\sum_{n=1}^{\infty}\left(\delta_{2 n}{ }^{\mathrm{I}} \sin \theta+\delta_{1 n}{ }^{\mathrm{I}} \cos \theta\right) \sin (n \pi \xi), \\
& \widetilde{w}^{\mathrm{I}}=\sum_{n=1}^{\infty}\left(-\delta_{1 n}{ }^{\mathrm{I}} \sin \theta+\delta_{2 n}{ }^{\mathrm{I}} \cos \theta\right) \sin (n \pi \xi),
\end{aligned}
$$

$$
\tilde{u}_{4}{ }^{\mathrm{I}}=\delta_{5}{ }^{\mathrm{I}}=\sum_{n=1}^{\infty} \delta_{5 n}{ }^{\mathrm{I}} \sin (n \pi \xi) \text {, }
$$

$\tilde{u}_{5}{ }^{\mathrm{I}}=\delta_{4}{ }^{\mathrm{I}}=\sum_{n=1}^{\infty} \delta_{4 n}{ }^{\mathrm{I}} \sin (n \pi \xi)$. 


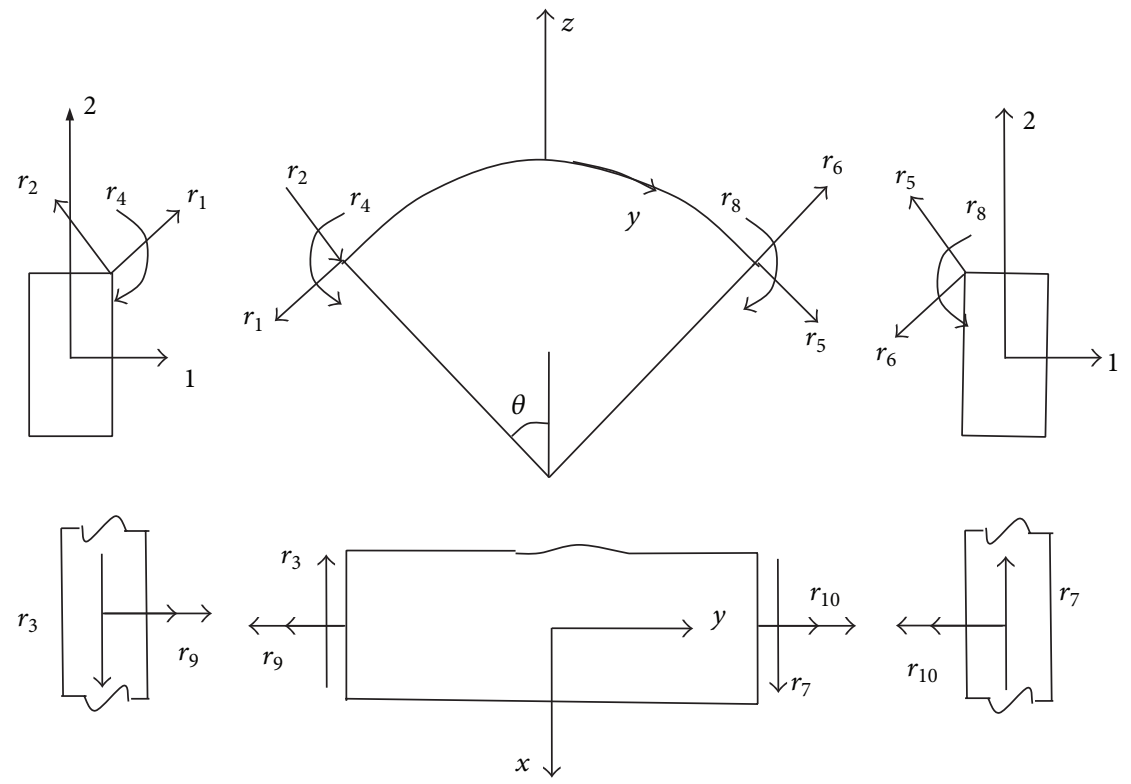

FIGURE 6: Edge forces distribution at the place where the shells are connected with the edge beam.

Edge II

Decompose $r_{5}, r_{6}$ along the axis 1 :

$-r_{5} \cos \theta-r_{6} \sin \theta=\sum_{n=1}^{\infty}\left(-r_{5 n} \cos \theta-r_{6 n} \sin \theta\right) \sin (n \pi \xi)$.

Decompose $r_{5}, r_{6}$ along the axis 2 :

$$
r_{5} \sin \theta-r_{6} \cos \theta=\sum_{n=1}^{\infty}\left(r_{5 n} \sin \theta-r_{6 n} \cos \theta\right) \sin (n \pi \xi) .
$$

According to Table 3, the displacement generated by the edge beam II can be denoted by

$$
\begin{gathered}
\delta_{1}{ }^{\mathrm{II}}=\sum_{n=1}^{\infty} \delta_{1 n}{ }^{\mathrm{II}} \sin (n \pi \xi), \quad \delta_{2}{ }^{\mathrm{II}}=\sum_{n=1}^{\infty} \delta_{2 n}{ }^{\mathrm{II}} \sin (n \pi \xi), \\
\delta_{3}{ }^{\mathrm{II}}=\sum_{n=1}^{\infty} \delta_{3 n}{ }^{\mathrm{II}} \cos (n \pi \xi) \quad \delta_{4}{ }^{\mathrm{II}}=\sum_{n=1}^{\infty} \delta_{4 n}{ }^{\mathrm{II}} \sin (n \pi \xi), \\
\delta_{5}{ }^{\mathrm{II}}=\sum_{n=1}^{\infty} \delta_{5 n}{ }^{\mathrm{II}} \sin (n \pi \xi) .
\end{gathered}
$$

Thus, the subdisplacement and angle of rotation of edge beam II in $u, v$, and $w$ directions are

$$
\begin{aligned}
& \tilde{u}_{1}^{\mathrm{II}}=\delta_{3}{ }^{\mathrm{II}}=\sum_{n=1}^{\infty} \delta_{3 n}{ }^{\mathrm{II}} \cos (n \pi \xi), \\
& \tilde{u}_{2}^{\mathrm{II}}=\sum_{n=1}^{\infty}\left(-\delta_{2 n}{ }^{\mathrm{II}} \sin \theta+\delta_{1 n}{ }^{\mathrm{II}} \cos \theta\right) \sin (n \pi \xi), \\
& \widetilde{w}^{\mathrm{II}}=\sum_{n=1}^{\infty}\left(\delta_{1 n}{ }^{\mathrm{II}} \sin \theta+\delta_{2 n}{ }^{\mathrm{II}} \cos \theta\right) \sin (n \pi \xi), \\
& \widetilde{u}_{4}^{\mathrm{II}}=\delta_{5}{ }^{\mathrm{II}}=\sum_{n=1}^{\infty} \delta_{5 n}{ }^{\mathrm{II}} \sin (n \pi \xi), \\
& \widetilde{u}_{5}^{\mathrm{II}}=\delta_{4}^{\mathrm{II}}=\sum_{n=1}^{\infty} \delta_{4 n}{ }^{\mathrm{II}} \sin (n \pi \xi) .
\end{aligned}
$$

The expression for shell's displacements is

$$
\begin{gathered}
\widetilde{u}_{1}=\sum_{n=1}^{\infty} \widetilde{u}_{1 n} \cos (n \pi \xi), \quad \widetilde{u}_{2}=\sum_{n=1}^{\infty} \widetilde{u}_{2 n} \sin (n \pi \xi), \\
\widetilde{w}=\sum_{n=1}^{\infty} \widetilde{w}_{n} \sin (n \pi \xi), \quad \widetilde{u}_{4}=\sum_{n=1}^{\infty} \widetilde{u}_{4 n} \cos (n \pi \xi), \\
\widetilde{u}_{5}=\sum_{n=1}^{\infty} \widetilde{u}_{5 n} \sin (n \pi \xi) .
\end{gathered}
$$

Then, shell's displacement on the edge is as follows. 
Edge I is

$$
\begin{aligned}
& \left.\tilde{u}_{1 q}^{\mathrm{I}}\right|_{\theta=-\theta_{0}}=\left.\tilde{u}_{1}\right|_{\theta=-\theta_{0}}=\left.\sum_{n=1}^{\infty} \tilde{u}_{1 n}\right|_{\theta=-\theta_{0}} \cos (n \pi \xi), \\
& \left.\tilde{u}_{2 q}^{\mathrm{I}}\right|_{\theta=-\theta_{0}}=\left.\widetilde{u}_{2}\right|_{\theta=-\theta_{0}}=\left.\sum_{n=1}^{\infty} \tilde{u}_{2 n}\right|_{\theta=-\theta_{0}} \sin (n \pi \xi), \\
& \left.\widetilde{w}_{q}^{\mathrm{I}}\right|_{\theta=-\theta_{0}}=\left.\widetilde{w}\right|_{\theta=-\theta_{0}}=\left.\sum_{n=1}^{\infty} \widetilde{w}_{n}\right|_{\theta=-\theta_{0}} \sin (n \pi \xi), \\
& \left.\tilde{u}_{4 q}{ }^{\mathrm{I}}\right|_{\theta=-\theta_{0}}=\left.\widetilde{u}_{4}\right|_{\theta=-\theta_{0}}=\left.\sum_{n=1}^{\infty} \widetilde{u}_{4 n}\right|_{\theta=-\theta_{0}} \cos (n \pi \xi), \\
& \left.\tilde{u}_{5 q}^{\mathrm{I}}\right|_{\theta=-\theta_{0}}=\left.\widetilde{u}_{5}\right|_{\theta=-\theta_{0}}=\left.\sum_{n=1}^{\infty} \widetilde{u}_{5 n}\right|_{\theta=-\theta_{0}} \sin (n \pi \xi) .
\end{aligned}
$$

Edge II is

$$
\begin{aligned}
& \left.\widetilde{u}_{1 q}^{\mathrm{II}}\right|_{\theta=\theta_{0}}=\left.\widetilde{u}_{1}\right|_{\theta=\theta_{0}}=\left.\sum_{n=1}^{\infty} \tilde{u}_{1 n}\right|_{\theta=\theta_{0}} \cos (n \pi \xi), \\
& \left.\tilde{u}_{2 q}^{\mathrm{II}}\right|_{\theta=\theta_{0}}=\left.\widetilde{u}_{2}\right|_{\theta=\theta_{0}}=\left.\sum_{n=1}^{\infty} \tilde{u}_{2 n}\right|_{\theta=\theta_{0}} \sin (n \pi \xi), \\
& \left.\widetilde{w}_{q}^{\mathrm{II}}\right|_{\theta=\theta_{0}}=\left.\widetilde{w}\right|_{\theta=\theta_{0}}=\left.\sum_{n=1}^{\infty} \widetilde{w}_{n}\right|_{\theta=\theta_{0}} \sin (n \pi \xi), \\
& \left.\widetilde{u}_{4 q}^{\mathrm{II}}\right|_{\theta=\theta_{0}}=\left.\widetilde{u}_{4}\right|_{\theta=\theta_{0}}=\left.\sum_{n=1}^{\infty} \widetilde{u}_{4 n}\right|_{\theta=\theta_{0}} \cos (n \pi \xi), \\
& \left.\widetilde{u}_{5 q}^{\mathrm{II}}\right|_{\theta=\theta_{0}}=\left.\widetilde{u}_{5}\right|_{\theta=\theta_{0}}=\left.\sum_{n=1}^{\infty} \widetilde{u}_{5 n}\right|_{\theta=\theta_{0}} \sin (n \pi \xi) .
\end{aligned}
$$

By using the continuous conditions of displacements to compare (46a)-(46e), (51a)-(51e), (49a)-(49e), and (52a)(52e), respectively, the displacement boundary conditions of shells can be obtained as follows.

Edge I is

$$
\begin{gathered}
\left.u_{1 n}\right|_{\theta=-\theta_{0}}=\delta_{3 n}{ }^{\mathrm{I}}, \\
\left.u_{2 n}\right|_{\theta=-\theta_{0}}=\delta_{2 n}{ }^{\mathrm{I}} \sin \theta+\delta_{1 n}{ }^{\mathrm{I}} \cos \theta, \\
\left.w_{n}\right|_{\theta=-\theta_{0}}=-\delta_{1 n}{ }^{\mathrm{I}} \sin \theta+\delta_{2 n}{ }^{\mathrm{I}} \cos \theta, \\
\left.u_{4 n}\right|_{\theta=-\theta_{0}}=\left.\delta_{5 n}{ }^{\mathrm{I}} \quad u_{5 n}\right|_{\theta=-\theta_{0}}=\delta_{4 n}{ }^{\mathrm{I}} .
\end{gathered}
$$

Edge II is

$$
\begin{gathered}
\left.u_{1 n}\right|_{\theta=\theta_{0}}=\delta_{3 n}{ }^{\mathrm{II}}, \\
\left.u_{2 n}\right|_{\theta=\theta_{0}}=-\delta_{2 n}{ }^{\mathrm{II}} \sin \theta+\delta_{1 n}{ }^{\mathrm{II}} \cos \theta, \\
\left.w_{n}\right|_{\theta=\theta_{0}}=-\delta_{1 n}^{\mathrm{II}} \sin \theta+\delta_{2 n}{ }^{\mathrm{II}} \cos \theta, \\
\left.u_{4 n}\right|_{\theta=\theta_{0}}=\delta_{5 n}^{\mathrm{II}},\left.\quad u_{5 n}\right|_{\theta=\theta_{0}}=\delta_{4 n}{ }^{\mathrm{II}} .
\end{gathered}
$$

According to the join condition, the ten undetermined coefficients can be solved, and trigonometric series expressions of displacements, angles of rotation, internal forces, and moments can be further obtained, whose changing curves can be drawn with MATLAB program.

\section{Numerical Examples}

Example 1. With the cylindrical shells roof that has been widely used in practical engineering as an example, assume the shells and edge beams are steel reinforcement and concrete materials. The calculating parameters are as follows: elastic modulus is $E=2.6 \times 10^{10} \mathrm{~Pa}$; Poisson's ratio is $v=0.167$; shells radius is $R=10 \mathrm{~m}, \alpha_{1}=5 / 6$, and $\alpha_{2}=0.06$; open angle is $2 \theta_{0}=\pi / 3$; widths of edge beam are $b=1.2 \mathrm{~m}, \alpha_{3}=1.5$, and $\alpha_{4}=0.1$; and the load is $q=-1 \times 10^{3} \mathrm{~N} / \mathrm{m}^{2}$. Through MATLAB program [15], the displacement and internal force of cylindrical shells are calculated, and by comparing the calculated results with the corresponding values of fixed edges and simply supported edges calculated by literatures [16], we get the following results as shown in Figure 7.

It can be seen from Figure 7 that, in the same load and dimensions of shells, the displacement of ribbed shells is bigger than that of fixed edges while it is smaller than that of simply supported edges; namely, the displacement of ribbed shells is between these of fixed edges and simply supported edges; the bending moments $M_{1}$ and $M_{2}$ attain their maximum value when they are near the ends, while the value is bigger than that of fixed edge and is smaller than that of simply supported edge when it is near the middle position. This change conforms to the basic mechanical laws.

Example 2. With the same calculating parameters (including dimensions of shells and edge beam and the applied loads) of Example 1, the displacement with different values of $\alpha_{1}$ and $\alpha_{2}$ is obtained, as shown in Figures 8 and 9.

Generally speaking, as seen in Figures 8 and 9, considering the shells radius is unchanged, the influence of transverse shear deformation on displacement increases gradually with the increase of $\alpha_{1}$ and $\alpha_{2}$.

\section{Conclusions}

Based on FSDT considering the effect of transverse shear, the displacement-based differential equation set for general problems of moderately thick cylindrical shells is obtained; the problem is simplified into a solvable eighth-order ordinary differential equation and a second-order ordinary differential equation by introducing four displacement functions.

In consideration of the effect of edge beams, the displacement of edge beams under edge force is obtained by using the given displacement differential equation of beams, and the displacement of edge beams is converted into the shells coordinate system. The join condition of moderately thick cylindrical ribbed shells is established according to the continuity of displacement. By using this join condition, the ten undetermined constants the displacement function 


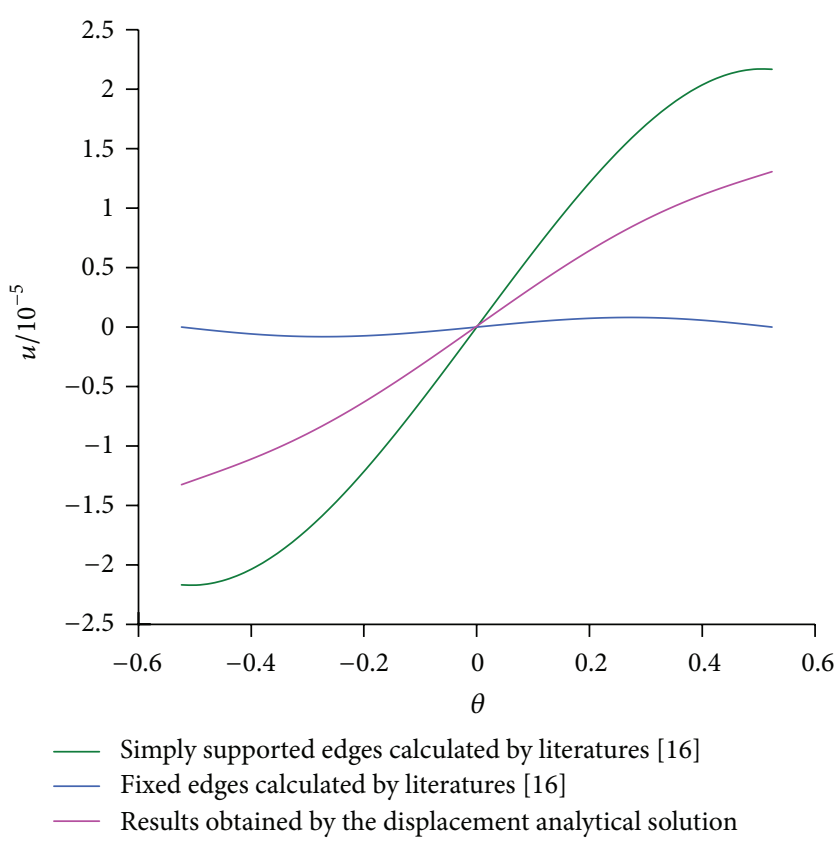

(a)

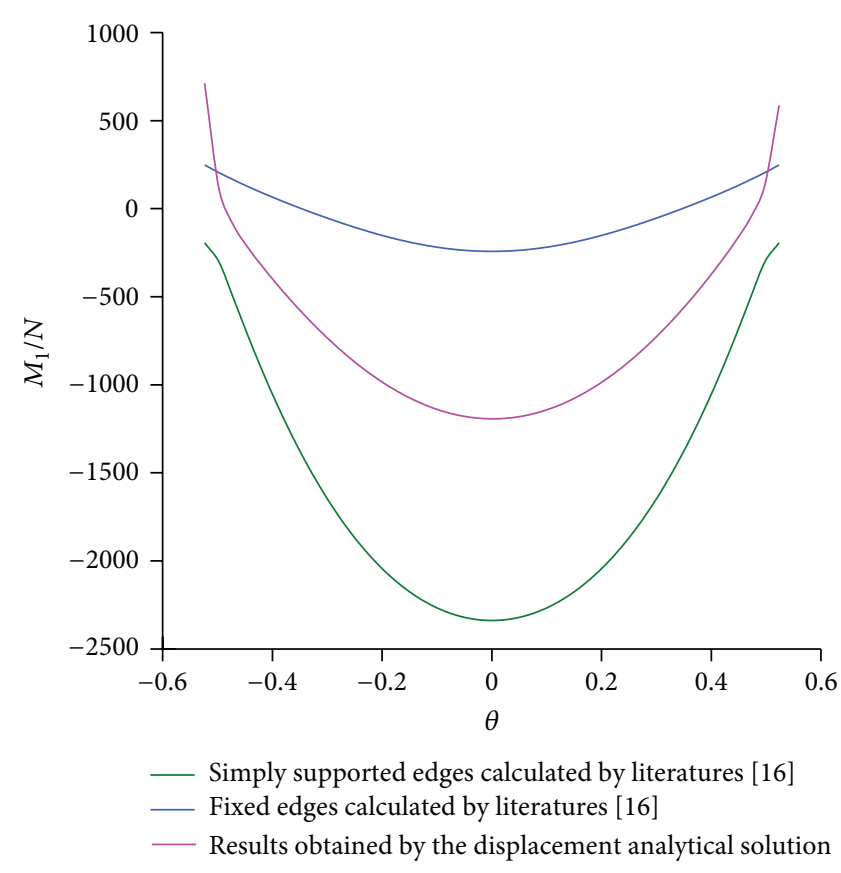

(c)

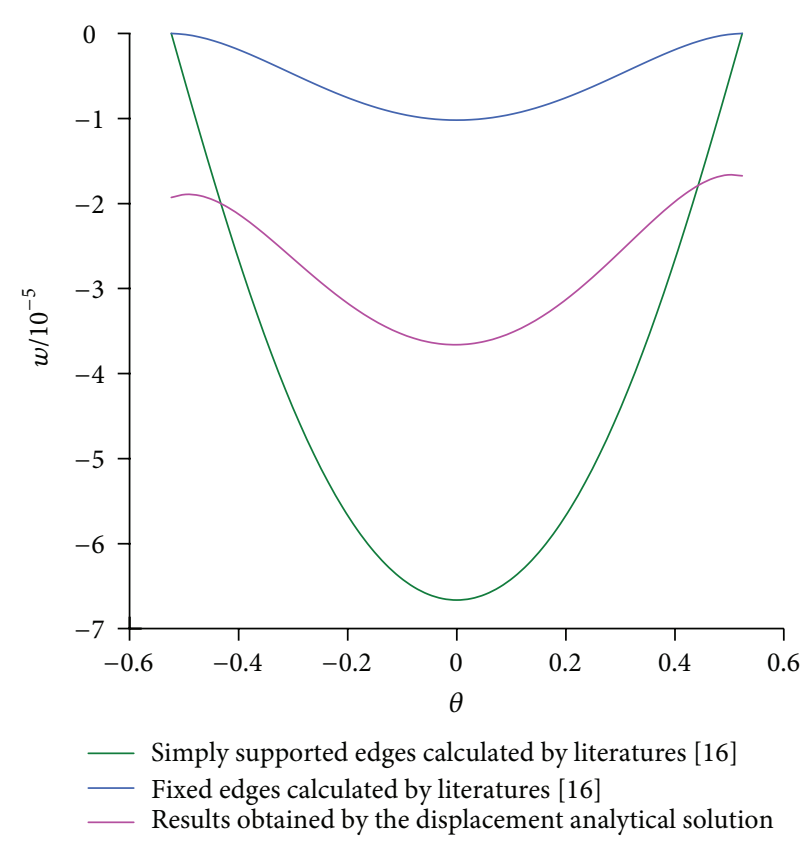

(b)

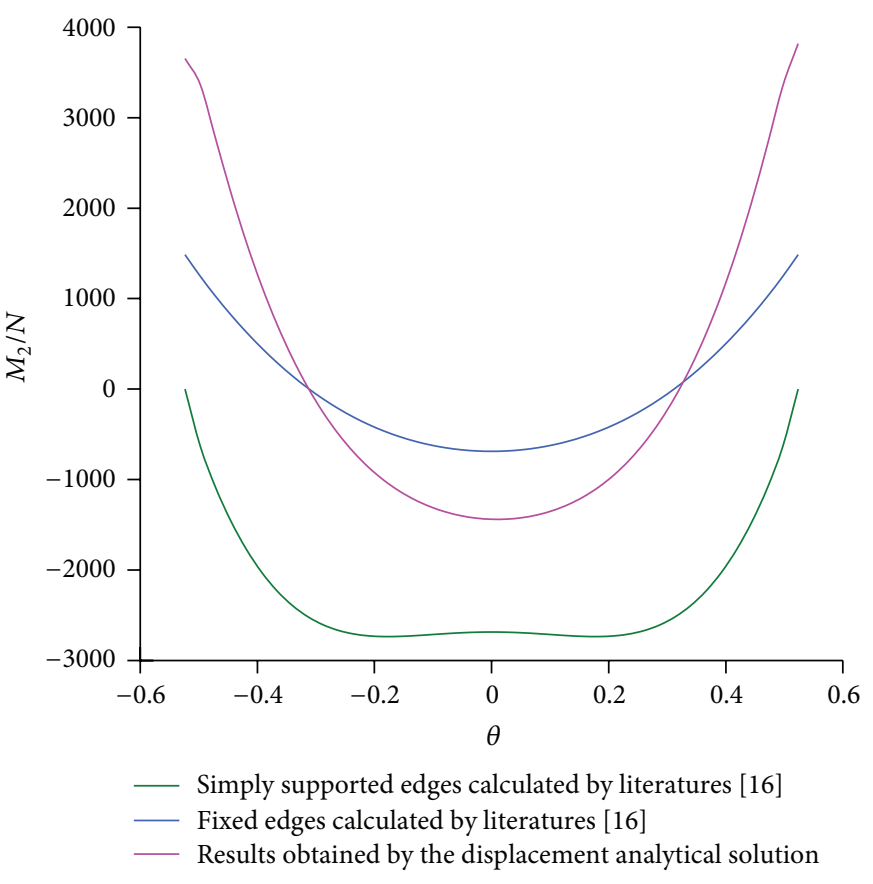

(d)

FIgURE 7: Comparison of the displacement and internal force under three boundary conditions.

contains are solved, and thus the solution of bending problem of moderately thick cylindrical ribbed shells is obtained. In addition, by comparing the displacement, internal force of ribbed shells with the displacement, internal force of fixed edges, and simply supported edges, the numerical results show that the displacement and internal force of ribbed shells are between these of fixed edges and simply supported edges, which explains that the solving methods proposed and the join condition established in this paper are correct.
For ease of the calculation, the first-order model considering the effect of transverse shear is adopted in this paper. The basic equations derived by using the above model are simpler, but they cannot fully satisfy the edge condition that the shear stress is 0 on the free surface, which is inconsistent with the actual situation and is also the defect-existing in the firstorder model itself. To remedy this defect, the higher-order model can be adopted for calculation in the follow-up study, so that the calculation results can get closer to the actual. 


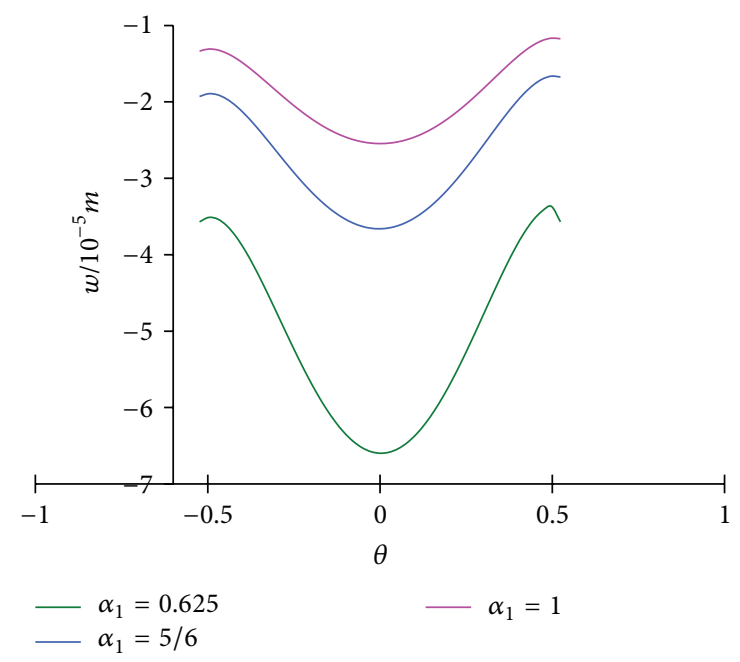

FIGURE 8: Comparison of the displacement with different values of $\alpha_{1}$.

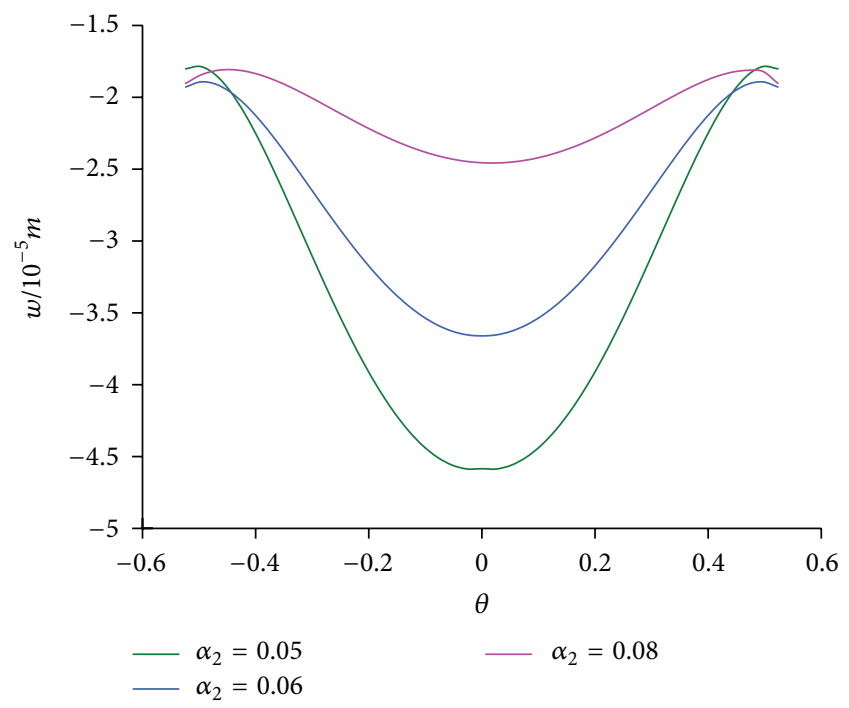

FIGURE 9: Comparison of the displacement with different values of $\alpha_{2}$.

\section{Conflict of Interests}

The authors declare that there is no conflict of interests regarding the publication of this paper.

\section{Acknowledgments}

This work is partially supported by National Natural Science Foundations of China (Grant no. 51478044). The authors also gratefully acknowledge the helpful comments and suggestions of the reviewers, which have improved the presentation.

\section{References}

[1] S. P. Timoshenko and S. Woinowosky-Krieger, Theory of Plates and Shells, McGraw-Hill, New York, NY, USA, 1959.
[2] W. Flugge, Stresses in Shells, Springer, Berlin, Germany, 1973.

[3] S. P. Timoshenko and J. N. Goodier, Theory of Elasticity, McGraw-Hill, New York, NY, USA, 1970.

[4] P. E. Tovstik, Stability of Thin Shells, Nauka, Moscow, Russia, 1995.

[5] G. Y. Jin, T. Ye, X. L. Ma, Y. Chen, Z. Su, and X. Xie, "A unified approach for the vibration analysis of moderately thick composite laminated cylindrical shells with arbitrary boundary conditions," International Journal of Mechanical Sciences, vol. 75, pp. 357-376, 2013.

[6] Y. Huang, H. R. Huang, and F. S. He, The Linear Theory of Elastic Shells, Science Press, Beijing, China, 2007.

[7] M. Di Sciuva, "An improved shear-deformation theory for moderately thick multilatered anisotropic shells and plates," Journal of Applied Mechanics, Transactions ASME, vol. 54, no. 3, pp. 589-596, 1987.

[8] N. N. Huang, "Influence of shear correction factors in the higher order shear deformation laminated shell theory," International Journal of Solids and Structures, vol. 31, no. 9, pp. 1263-1277, 1994.

[9] A. Tabiei and G. Simitses, "Imperfection sensitivity of shear deformable moderately thick laminated cylindrical shells," Computers \& Structures, vol. 62, no. 1, pp. 165-174, 1997.

[10] R. A. Chaudhuri and K. R. Abu-Arja, "Static analysis of moderately-thick finite antisymmetric angle-ply cylindrical panels and shells," International Journal of Solids and Structures, vol. 28, no. 1, pp. 1-15, 1991.

[11] A. Chakrabarti, B. Mukhopadhyay, and R. K. Bera, "Nonlinear stability of a shallow unsymmetrical heated orthotropic sandwich shell of double curvature with orthotropic core," International Journal of Solids and Structures, vol. 44, no. 16, pp. 5412-5424, 2007.

[12] C. O. Horgan and J. K. Knowles, "Recent developments concerning Saint-Venant's principle," Advances in Applied Mechanics, vol. 23, pp. 179-269, 1983.

[13] M. Khalifa Ahmed, "Elastic buckling behaviour of a fourlobed cross section cylindrical shell with variable thickness under non-uniform axial loads," Mathematical Problems in Engineering, vol. 2009, Article ID 829703, 17 pages, 2009.

[14] W.-Y. Jung and S.-C. Han, "An 8-node shell element for nonlinear analysis of shells using the refined combination of membrane and shear interpolation functions," Mathematical Problems in Engineering, vol. 2013, Article ID 276304, 16 pages, 2013.

[15] The MathWorks Inc, MATLAB Getting Started Guide, The MathWorks Inc, 2009.

[16] Y. Huang, C.-X. Guo, and Y.-Y. Wang, "Displacement solution of medium cylindrical shells' bending problem," Journal of Xian University of Architecture and Technology, vol. 39, no. 6, pp. 746751, 2007. 


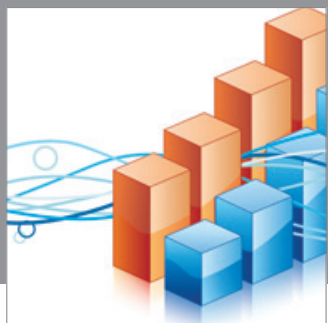

Advances in

Operations Research

mansans

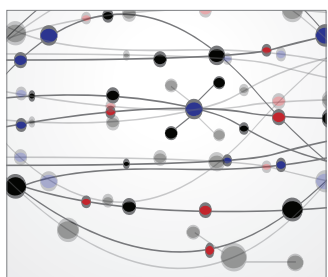

The Scientific World Journal
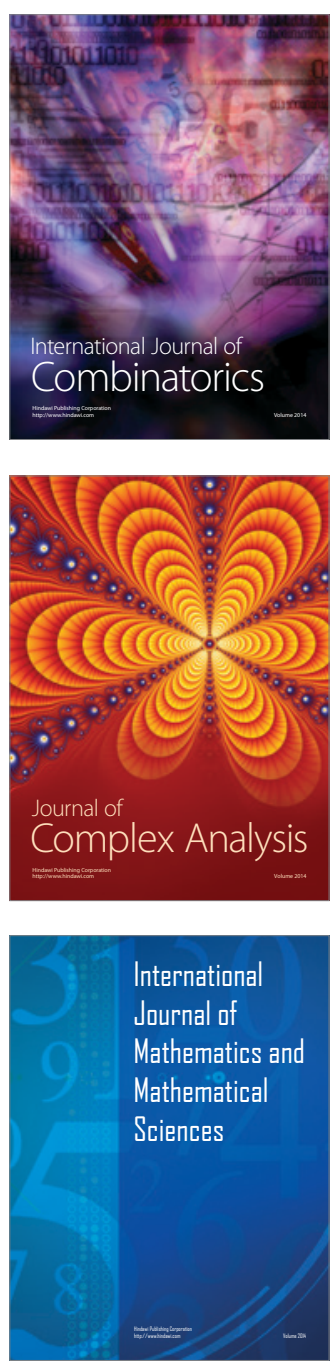
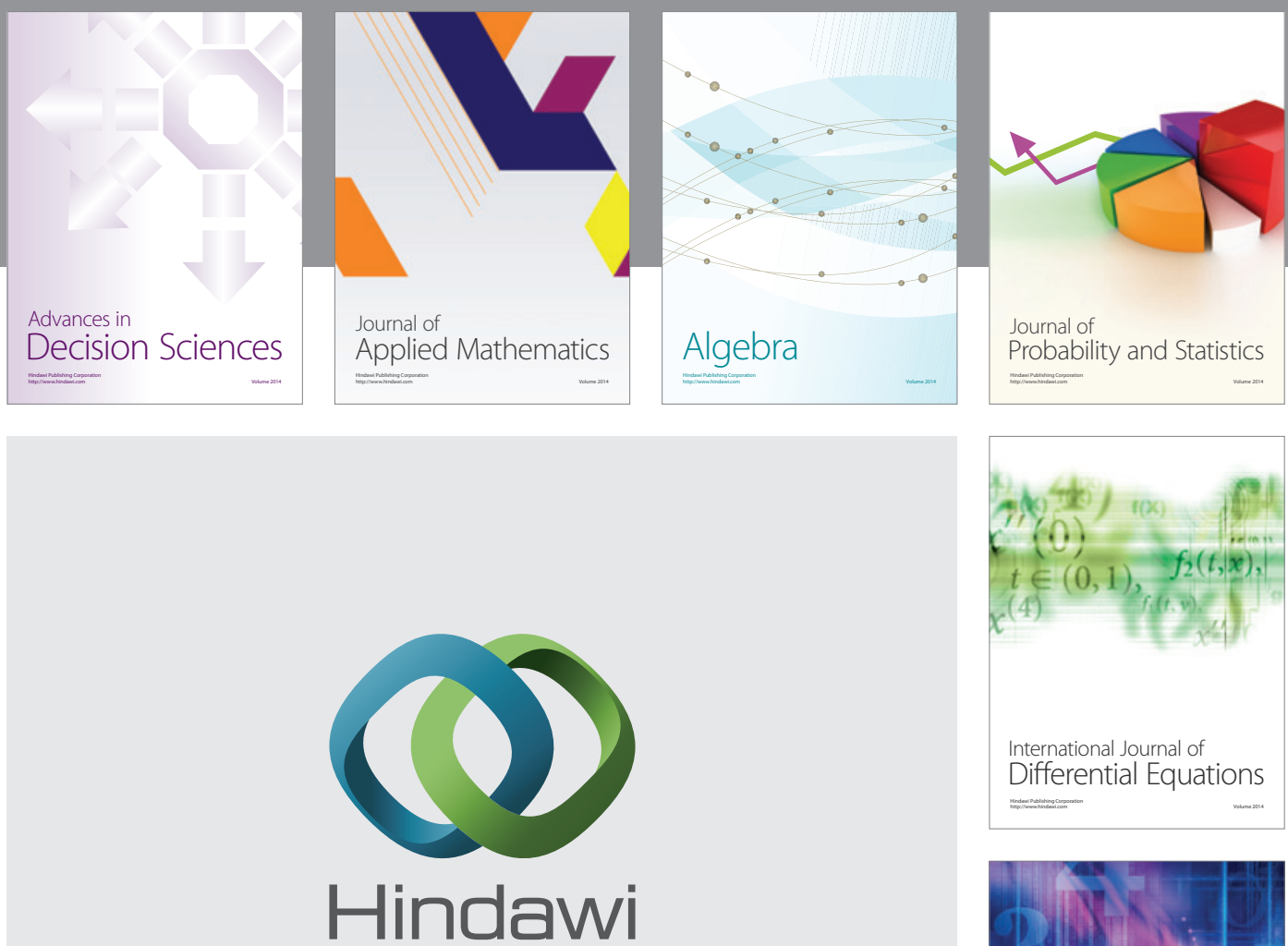

Submit your manuscripts at http://www.hindawi.com
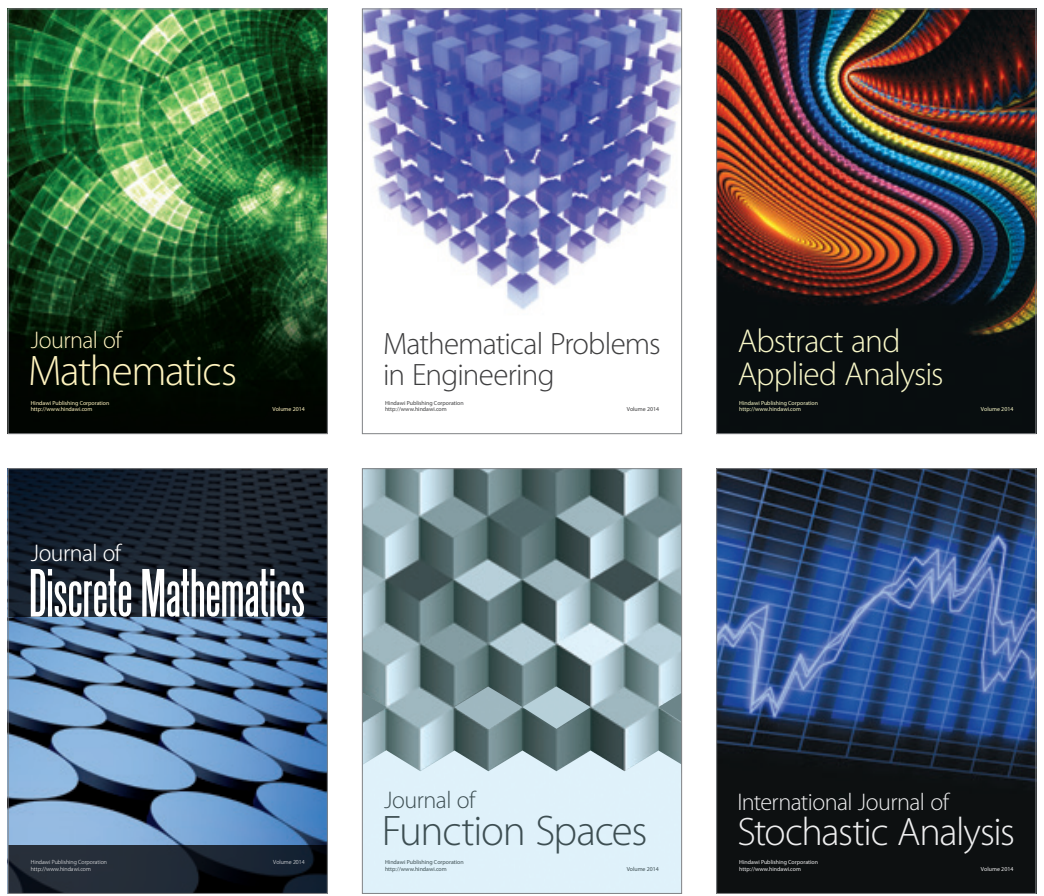

Journal of

Function Spaces

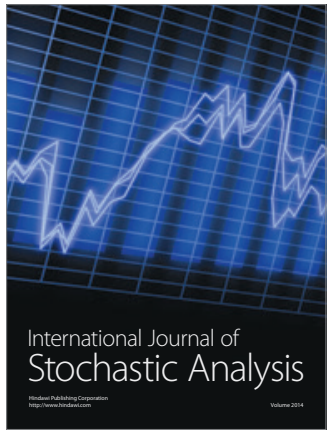

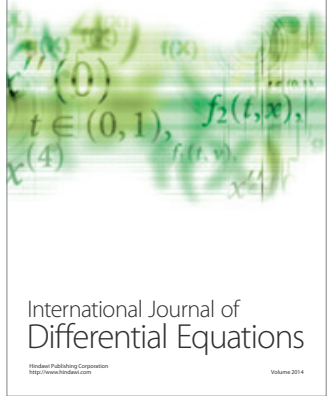
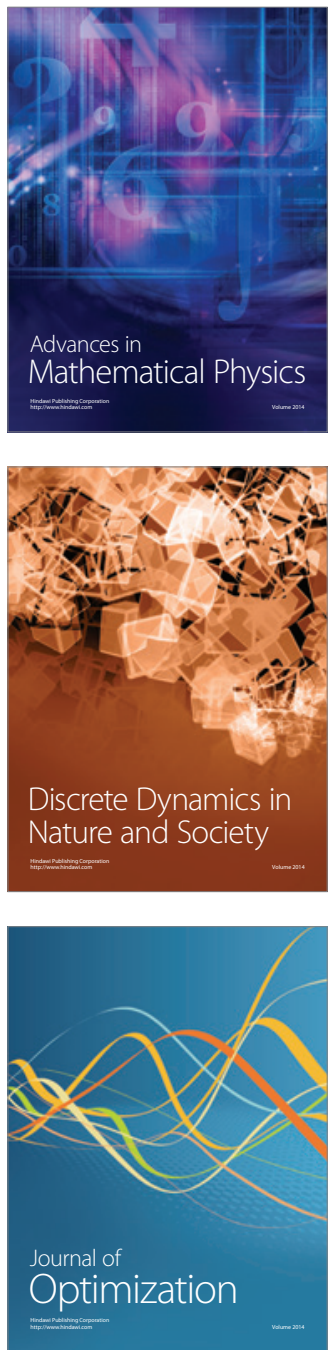